\title{
Anharmonic mid-infrared vibrational spectra of benzoic acid monomer and dimer
}

\author{
Jens Antony, ${ }^{1}$ Gert von Helden, ${ }^{2, \text { ศ }}$ Gerard Meijer, ${ }^{2}$ and Burkhard Schmidt ${ }^{1,}{ }^{\dagger}$ \\ ${ }^{1}$ Freie Universität Berlin, Institute of Mathematics II, \\ Arnimallee 2-6, D-14195 Berlin, Germany \\ ${ }^{2}$ Fritz-Haber-Institut der Max-Planck-Gesellschaft, \\ Faradayweg 4-6, D-14195 Berlin, Germany
}

\begin{abstract}
Anharmonic vibrational calculations for the benzoic acid monomer and dimer in the mid-IR regime $\left(500 \ldots 1800 \mathrm{~cm}^{-1}\right)$ are reported. Harmonic frequencies and intensities are obtained at the DFT/B3LYP level of theory employing D95(d,p) and cc-pVTZ basis sets. Anharmonic corrections obtained from standard perturbation theory lead to red shifts of $1 \ldots 3 \%$. In almost all cases, the resulting frequencies deviate by less than $1 \%$ from previous measurements [J. Chem. Phys. 119, 11180 (2003)]. Calculated intensities are in qualitative agreement with the absorption experiment, with the cc-pVTZ values being superior to the D95(d,p) ones for a few modes of the dimer. The antisymmetric out-of-plane bending mode of the dimer, which is strongly blue-shifted with respect to the monomer frequency, represents a remarkable exception: The harmonic frequencies obtained for the two basis sets differ notably from each other, and the anharmonically corrected frequencies deviate from the experimental value by $8 \%(\mathrm{D} 95(\mathrm{~d}, \mathrm{p}))$ or $3 \%$ (cc-pVTZ). Non-perturbative calculations in reduced dimensionality reveal that the relatively small total anharmonic shift (few tens of $\mathrm{cm}^{-1}$ ) comprises of partly much larger contributions (few hundreds of $\mathrm{cm}^{-1}$ ) which are mostly canceling each other. Many of the individual anharmonic couplings are beyond the validity of second order perturbation theory based on cubic and semi-diagonal quartic force constants only. This emphasizes the need for high-dimensional, non-perturbative anharmonic calculations at high quantum chemical level when accurate frequencies of $\mathrm{H}$-atom vibrations in double hydrogen bonds is sought for.
\end{abstract}

*Electronic address: helden@fhi-berlin.mpg.de

${ }^{\dagger}$ Electronic address: burkhard@math.fu-berlin.de 


\section{INTRODUCTION}

Complexes dominated by hydrogen bonds play a central role in many fields of chemistry. In particular, double hydrogen bonds are of paramount importance in biochemistry because of their abundance in nucleic acid base pairs holding together the double stranded helices in DNA. Carboxylic acid dimers provide the simplest model systems to study doubly hydrogen bonded systems where the cyclic arrangement of these dimers gives rise to a symmetric double proton transfer mediated by concerted tunneling [1, 2, 3]. Vibrational spectroscopy of these complexes has proven especially useful in elucidating structure and dynamics of these complexes. In particular, there has been an increasing interest in gas phase vibrational spectroscopy of these aggregates in recent years. Due to the availability of double resonance spectroscopy (ion dip technique), the benzoic acid dimer has received considerable attention in recent years [4, 5, 6]. It is well known that the strongest effects of the intermolecular bonding can be found in the region of the $\mathrm{OH}$ stretching frequency $\left(\geq 2500 \mathrm{~cm}^{-1}\right)$. In addition to a considerable frequency shift with respect to the monomeric vibration, very broad structures have been observed in the spectra of carboxylic acid dimers, extending over several hundreds of $\mathrm{cm}^{-1}$ [4, 7]. Similar features were also found for related dimers such as $\mathrm{CH}_{3} \mathrm{NO}_{2}^{-} \cdot \mathrm{H}_{2} \mathrm{O}$ and $\mathrm{CH}_{3} \mathrm{CO}_{2}^{-} \cdot \mathrm{H}_{2} \mathrm{O}[8]$. However, also the mid-infrared (IR) spectrum (500$2500 \mathrm{~cm}^{-1}$ ) is providing a wealth of information on strongly hydrogen bonded systems in the gas phase as has been demonstrated in spectroscopic studies of the protonated water dimer [9, 10] and dimers of ethers [11] or even nucleobases [12]. In a recent study of the benzoic acid monomer and dimer, an inspection of a larger portion of the mid-infrared spectrum has been carried out [6]. Also in that frequency regime, quite a few resonances of the dimer spectrum were found to be distinctly different from those of the monomer. The extreme case is the strong blue shift of the $\mathrm{COH}$ out-of-plane bending vibration from $571 \mathrm{~cm}^{-1}$ in the monomer to $962 \mathrm{~cm}^{-1}$ in the dimer which is essentially caused by an effective stiffening of the bending motion due to hydrogen bonding. Note that also in an argon matrix an almost identical value $\left(960 \mathrm{~cm}^{-1}\right)$ was found [13].

With the availability of powerful computers and the advent of efficient density functional theory (DFT) methods implemented in standard codes, structure and dynamics of systems containing a few tens of atoms (or even more) are now within reach. Vibrational spectra of small molecules of biological or pharmaceutical relevance are routinely treated combing 
DFT electronic structure calculations with a harmonic analysis [14]. Due to deficiencies of the quantum chemical calculations and/or due to the neglect of anharmonic effects, these frequencies are typically a few percent higher than the observed ones. However, using empirical scaling factors, satisfactory quantitative agreement with experimental vibrational spectra in the mid-IR regime can be achieved in many cases [15]. Nonetheless, pronounced discrepancies are occasionally found, e. g., for vibrational modes where the contributing atoms are directly involved in a hydrogen bond. For the case of the benzoic acid monomer and dimer to be studied here, the situation is as follows [6]: Employing DFT (B3LYP) calculations with a basis set of moderate size (D95(d,p)), almost all of the observed midIR vibrational frequencies can be reproduced within the harmonic approximation (using an empirical scaling factor of $98 \%$ ). However, the $\mathrm{COH}$ bending vibrations of the dimer exhibit a peculiarity. While the in-plane vibration can be reproduced by that approach very well, the theoretical value for the (antisymmetric) out-of-plane bending mode is $7 \%$ higher than the observed one [6]. A similar situation was also found for the formic acid dimer [5]. The main goal of the present work is a quantitative explanation of this discrepancy. In particular, the question has to be answered whether this is due to insufficient quality of the quantum chemical treatment or due to the use of the harmonic approximation for the molecular vibrations (or both). At least for the case of the $\mathrm{OH}$ stretch spectra of carboxylic acid dimers, the importance of anharmonic effects has been demonstrated. Theoretical models involving strong anharmonic coupling to several other vibrational modes can qualitatively reproduce the unusual features of the measured spectra in the $3000 \mathrm{~cm}^{-1}$ region [4, 5]. Similarly, large anharmonic coupling constants were found in a far-infrared study of the acetic acid dimer as well [16]. However, these investigations already show the principle problem encountered in simulations of anharmonic vibrational spectra of medium-sized to large molecular systems. On the one hand there are highly developed, very precise methods based on variational calculus which are, however, essentially limited to few degrees of freedom only [17]. On the other hand, the standard perturbation based approach to anharmonic vibrations is computationally less demanding but its range of validity is not quite clear [18, 19, 20, 21, 22]. The recently developed vibrational self-consistent-field techniques [23] and its extensions [24, 25, 26] appear to provide a promising compromise.

In the present work we focus on a quantitative assessment of anharmonic effects on the $\mathrm{COH}$ out-of-plane vibrational frequency of benzoic acid. By comparison of monomer and 
dimer vibrations we aim at shedding some light on the effect of the strong double hydrogen bond. For this purpose we calculate anharmonic vibrational frequencies following a mixed strategy. First, perturbative calculations are carried out in full dimensionality. Based on these results, we then concentrate on the most important anharmonic couplings which are treated in a non-perturbative manner but in reduced dimensionality. The remainder of the paper is organized as follows. Sec. II explains the methods used for the calculation of electronic structure and of harmonic and anharmonic vibrational frequencies. The results of perturbative and non-perturbative vibrational analysis of the benzoic acid monomer and dimer are discussed in Sec. III. Finally, our conclusions are presented in Sec. IV.

\section{METHODS}

\section{A. Electronic structure calculations}

Quantum chemical calculations were performed for the benzoic acid monomer and dimer within the framework of density functional theory (DFT) using Becke's three parameter Lee-Yang-Pair (B3LYP) functional [27], as implemented in the Gaussian software [28]. Both Dunning's D95(d,p) [29] and the correlation consistent cc-pVTZ basis set [30] were used comprising 174 or 354 basis functions for each of the benzoic acid moieties, respectively. Normal mode vibrational amplitudes, harmonic frequencies, and infrared intensities of molecular vibration were calculated based on an analytic evaluation of the Hessian matrix. For the monomer the geometry is planar $\left(C_{s}\right.$ symmetry); for the dimer a planar structure with cyclic arrangement of the hydrogen bonds $\left(C_{2 h}\right.$ symmetry) was obtained. These structures were found to be global minima on their potential energy surfaces; no imaginary frequencies were found in the vibrational analysis.

While it would be desirable to check the accuracy of the density functional theory against high-level wave-function (WV) based electronic structure calculations, the numerical effort, especially for the benzoic acid dimer, is exceedingly high. However, for the closely related formic acid monomer and dimer a systematic study of optimized geometries and harmonic frequencies was conducted comparing DFT and WV based methods for several basis sets [5]. With the largest basis set considered there (aug-cc-pVTZ), B3LYP and MP2 calculations yield very good agreement for all vibrational modes of the formic acid monomer. While 
the high frequency modes $(\mathrm{CH}, \mathrm{OH}$ stretching) of the formic acid dimer differ by up to $5 \%$, the mid-IR vibrational modes were found to differ by less than $1 \%$. For example, the calculated harmonic frequencies for the out-of-plane $\mathrm{COH}$ bending mode are $995 \mathrm{~cm}^{-1}$ and $1002 \mathrm{~cm}^{-1}$ for the MP2 and B3LYP method, respectively [5]. Moreover, no tendency towards a reduction of the deviation from the experimental value of $917 \mathrm{~cm}^{-1}$ can be deduced for increasing basis set. Similarly, a discrepancy of $6 \%$ was found for the $\mathrm{COH}$ bending wavenumber of the benzoic acid dimer employing a mixed basis set similar to the D95(d,p) basis used in this work, while larger basis sets were not considered in Ref. [5]. Hence, we conclude that for carboxylic acid dimer $\mathrm{COH}$ bending modes it is not immediately clear whether the quantum chemical method or anharmonic effects are mainly responsible for the mismatch between theory and experiment found in previous works [5, 6].

\section{B. Perturbation theory}

The standard approach to the calculation of anharmonic vibrational frequencies of a polyatomic molecule rests on an expansion of the potential energy hypersurface in terms of normal coordinates. The contribution of the anharmonic terms is then treated as a perturbation acting on the harmonic normal mode Hamiltonian. Vibrational energy levels characterized by a set of quantum numbers $\left\{n_{i}\right\}$ can be expressed as [18, 19, 20, 21, 22]

$$
\frac{E}{h c}=\sum_{r=1}^{3 N-6} \omega_{r}\left(n_{r}+\frac{1}{2}\right)+\sum_{r \geq s} \chi_{r s}\left(n_{r}+\frac{1}{2}\right)\left(n_{s}+\frac{1}{2}\right)
$$

where $\omega_{r}$ are the harmonic frequencies of vibration (in units of wavenumbers). Neglecting Coriolis effects, the diagonal and off-diagonal anharmonic constants $\chi$ are obtained from

$$
\begin{aligned}
\chi_{r r} & =\frac{1}{16} \phi_{r r r r}-\frac{1}{16} \sum_{s=1}^{3 N-6} \phi_{r r s}^{2} \frac{8 \omega_{r}^{2}-3 \omega_{s}^{2}}{\omega_{s}\left(4 \omega_{r}^{2}-\omega_{s}^{2}\right)} \\
\chi_{r s} & =\frac{1}{4} \phi_{r r s s}-\frac{1}{4} \sum_{t=1}^{3 N-6} \frac{\phi_{r r t} \phi_{t s s}}{\omega_{t}} \\
& -\frac{1}{2} \sum_{t=1}^{3 N-6} \frac{\phi_{r s t}^{2} \omega_{t}\left(\omega_{t}^{2}-\omega_{r}^{2}-\omega_{s}^{2}\right)}{\Delta_{r s t}}, \quad r \neq s \\
\Delta_{r s t} & =\left(\omega_{r}+\omega_{s}+\omega_{t}\right)\left(\omega_{r}-\omega_{s}-\omega_{t}\right) \\
& \times\left(-\omega_{r}+\omega_{s}-\omega_{t}\right)\left(-\omega_{r}-\omega_{s}+\omega_{t}\right)
\end{aligned}
$$

where $\phi_{r s t}$ and $\phi_{\text {rrss }}$ stand for mixed third and fourth derivatives of the potential energy function with respect to the normal coordinates of vibration. The above formulae have 
been implemented in the Gaussian 03 software thus making anharmonic calculations easily available to a wider range of the quantum chemical community [28]. While second derivatives are obtained analytically in Gaussian, third and fourth order terms are computed from finite differences with the effort scaling linearly with the number of normal modes [21, 22, 31]. We note that for several small molecules the quality of the computed anharmonic corrections has been shown to be not very sensitive to the choice of the quantum chemical method or the size of the basis set [22]. This finding opens the way for the efficient calculation of anharmonic vibrations where only harmonic frequencies are obtained from a high-level computations while cheaper models are sufficient to estimate the anharmonic corrections.

In applying the above procedure, one has to keep in mind that this approach rests on two assumptions: (1) In the vicinity of a minimum, the potential energy function can be expressed in terms of a Taylor series which is truncated to yield a fourth order polynomial. (2) The above formulae rest on first and second order perturbation theory (PT2) for the treatment of the quartic and cubic force constants, respectively. It is noted that the restriction to first order for the quartic contribution implies that only diagonal $\left(\phi_{r r r r}\right)$ and semi-diagonal $\left(\phi_{\text {rrss }}\right)$ derivatives are taken into account. While higher order treatments were explored for one-dimensional anharmonic oscillators [32, 33], no such approaches can be found in the literature for the multi-dimensional case of polyatomic molecular vibration.

However, it is not a priori clear whether these two assumptions are justified: As will be discussed below, some of the two- and three-dimensional slices of the potential energy function are more complicated functions of the respective normal coordinates. Moreover, the use of second order perturbation theory (PT2) is questionable in cases where the anharmonic effects are becoming large. In Sec. IIIB we are going to present examples where either one (or both) of the two assumptions are not valid.

\section{Non-perturbative calculations}

As an alternative we pursue non-perturbative anharmonic vibrational analysis. While variational treatments or the vibrational self-consistent-field approach can treat approximately up to ten degrees of freedom, for systems of the size considered here (15 or 30 atoms), studies in full dimensionality are clearly out of the range of today's computational means. Nevertheless, low-dimensional calculations for suitably chosen vibrational modes are instru- 
mental in gaining a deeper understanding of the anharmonic coupling mechanisms. In the present work we proceed as follows: On the basis of the harmonic vibrational analysis of benzoic acid monomer and dimer, one-, two-, and three-dimensional scans of the potential energy are performed with single point energy calculations with the equilibrium structure displaced along a single normal coordinate (1D) or along the linear combination of two or three of those coordinates $(2 \mathrm{D}, 3 \mathrm{D})$. The respective grid representations have a spacing of $0.1 \AA$ for hydrogen stretching vibrations, $0.2 \AA$ for the remaining intramolecular vibrations, and $0.5 \AA$ for the intermolecular vibrations of the dimer.

Eigenvalues and eigenfunctions corresponding to bound states are then obtained by propagating the nuclear Schrödinger equation in imaginary time where an expansion of the evolution operator in terms of Chebychev polynomials was used. The Hamiltonian is represented on an equidistant grid thus allowing the kinetic energy to be calculated by fast Fourier transforms [34, 35]. Vibrationally excited states were also obtained by this relaxation method, where the contribution of lower states have to be projected out at each evaluation of the Hamiltonian. The differences of ground and excited state energies yield fundamental frequencies. These calculations can be used to investigate the quality of the perturbation-based results: First, a comparison of the potential energy surfaces with low-order polynomials directly allows to judge the validity of the truncated polynomial expansion. Second, through a comparison of the non-perturbative results with corresponding one-, two-, and threedimensional perturbative calculations, the validity of the perturbation treatment (PT2) can be critically assessed.

\section{RESULTS AND DISCUSSION}

\section{A. Perturbative anharmonic vibrational analysis}

Monomer. Due to computational restrictions owing to the size of the molecules under investigation, we follow the above-mentioned mixed strategy of Ref. 22] throughout this work. We are employing a very large basis set for the computation of the harmonic frequencies while anharmonic corrections have to be estimated from cheaper calculations. First, infrared intensities and harmonic frequencies are calculated for the benzoic acid monomer $\left(C_{s}\right.$ symmetry) at the DFT (B3LYP) level of theory using the D95(d,p) [29] and the much 
larger cc-pVTZ [30] basis set. In addition, anharmonic contributions based on the perturbation formulae (1/2) are computed only for the smaller D95(d,p) basis set in full dimensionality and are used to correct both the D95(d,p) and the cc-pVTZ results. Our results given in Tab. I include only those vibrational modes that were observed in the previous gas phase infrared absorption spectroscopic study covering the mid-IR regime $500 \ldots 1800$ $\mathrm{cm}^{-1}[6$. Complete tables of force constants and frequencies are available from the authors [36]. Note that throughout the scope of this work normal modes are numbered according to ascending D95(d,p) frequencies. The harmonic vibrational frequencies are found to be lowered by $1 \ldots 3 \%$ owing to the effect of anharmonicity. This leads to excellent agreement with the experimental absorption spectrum of Ref. [6] with deviations in the frequencies rarely exceeding $1 \%$, in almost all cases even significantly below, see Fig. 1(a). Moreover, on grounds of the comparison with experiment, neither frequencies nor intensities provide clear preference for one of the basis sets used here. This indicates that the empirical scaling of the harmonic frequencies for the benzoic acid monomer used, e. g. in Refs. [6, 15], mainly reflects the effect of anharmonicity rather than deficiencies of the quantum-chemical method.

Dimer. Upon dimerization the number of intramolecular vibrational modes is doubled with the A' modes of the monomer ( $C_{s}$ symmetry) correlating with $A_{g}$ and $B_{u}$ modes of the dimer $\left(C_{2 h}\right.$ symmetry) and A" correlating with $A_{u}$ and $B_{g}$. In most cases, the dimer modes are best described as either symmetric (gerade) or anti-symmetric (ungerade) combinations of two monomeric vibrations where only the latter ones are infrared-active. Our results are summarized in Tab. II and in Fig. 1 (b): The frequency shifts with respect to the corresponding monomeric frequencies are largely different: While most frequencies are shifted by less than $10 \mathrm{~cm}^{-1}$ (modes 25, 27, 32, 44, 48, 53, 60, 63, 65, 67, 70), the modes involving atoms participating in the double hydrogen bond exhibit shifts of a few 10 $\mathrm{cm}^{-1}$ (modes 20, 24, 30, 49, 56, 58, 61, 72). For those modes also the splitting of gerade and ungerade modes is appreciable while in the other cases the splitting is often less than $1 \mathrm{~cm}^{-1}$. Out of the vibrational modes in the mid IR frequency regime considered here, the out-of-plane C-O-H bending mode experiences the largest effect of dimerization: Using the D95(d,p) basis set, the harmonic wavenumber is shifted (and split) from $594 \mathrm{~cm}^{-1}\left(\nu_{8}, \mathrm{~A}^{\prime}\right)$ in the monomer to $1003 \mathrm{~cm}^{-1}\left(\nu_{39}, \mathrm{~B}_{g}\right)$ and $1050 \mathrm{~cm}^{-1}\left(\nu_{46}, \mathrm{~A}_{u}\right)$ in the dimer. While all other frequencies do not change substantially upon increasing the basis size from D95(d,p) 
to cc-pVTZ, the latter case represents a remarkable exception: For the larger basis set (ccpVTZ) there are two intensely absorbing normal modes containing notable contribution of $\mathrm{COH}$ out-of-plane bending with frequencies of 1005 and $1008 \mathrm{~cm}^{-1}$ (both of $\mathrm{A}_{u}$ symmetry) thus being substantially lower than the corresponding result $\left(1050 \mathrm{~cm}^{-1}\right)$ for the smaller basis set $(\mathrm{D} 95(\mathrm{~d}, \mathrm{p}))$. Note that this is in contrast to the detailed investigation of the formic acid dimer in Ref. [5], where no pronounced dependence of the $\mathrm{COH}$ bending frequency on the basis set was found. This appears to indicate that the calculations are sensitive to the coupling between $\mathrm{COH}$ bending motion and ring $\mathrm{CCH}$ bending also present in the 1005 and $1008 \mathrm{~cm}^{-1}$ modes of the dimer.

Because of the enormous computational effort, anharmonic corrections in full dimensionality using Eqs. (1) 2) can only be evaluated for the D95(d,p) basis set. These corrections are used for both basis sets. As indicated in Tab. II] the anharmonic effects again lower the vibrational frequencies by about $1 \ldots 3 \%$. As in the case of the monomer, the agreement with measured gas phase frequencies [6] is excellent in almost all cases, with deviations being below $1 \%$. While the frequencies obtained for the two basis sets are in almost all cases very close to each other, the cc-pVTZ intensities are in some cases closer to the experimental ones, see Fig. 1 (b). However, there is a huge discrepancy for the frequency of the antisymmetric out-of-plane C-O-H bending mode $\left(\nu_{46}, \mathrm{~A}_{u}\right)$. The anharmonically corrected D95(d,p) value $\left(1037 \mathrm{~cm}^{-1}\right)$ differs by $8 \%$ from the measured gas phase value $\left(962 \mathrm{~cm}^{-1}\right)$ [6]. Also for the much larger cc-pVTZ basis, the anharmonic values of $992 / 995 \mathrm{~cm}^{-1}$ are still $3 \%$ higher than the measured one. Furthermore, similar findings were also made in previous work on the benzoic acid dimer, where an unusual discrepancy of measured and calculated values for the out-of-plane $\mathrm{COH}$ bending wavenumber was published in Ref. [5]. Although the present results indicate that the deviations found in previous works are partly an effect of the basis size, the remaining discrepancy may still be due to anharmonic effects. Hence, we shall present a more detailed study of anharmonic couplings of the $\nu_{8}$ mode of the monomer and the $\nu_{46}$ mode of the dimer in the following section.

\section{B. Non-perturbative vibrational analysis of uncoupled modes}

Monomer. As a first step towards a non-perturbative anharmonic vibrational analysis, we present results for anharmonicity arising from calculations for uncoupled normal 
modes. We restrict ourselves to those vibrational modes featuring a notable amplitude of the acid group hydrogen atom. Our calculated B3LYP/D95(d,p) frequencies of benzoic acid monomer are summarized in Tab. III. The anharmonic frequencies obtained from bound state calculations based on one-dimensional scans of the potential energy surface, see Sec. IIC. Note that the table includes only those modes where the contribution of anharmonicity exceeds $10 \mathrm{~cm}^{-1}$. The largest anharmonic effect is found for the $\mathrm{COH}$ out-of-plane bending frequency $\left(\nu_{8}\right)$ which is shifted by $173 \mathrm{~cm}^{-1}$ to the blue while the umbrella mode $\left(\nu_{12}\right)$ which involves similar $\mathrm{H}$ atom motion as the $\nu_{8}$ mode is blue-shifted by only $12 \mathrm{~cm}^{-1}$ due to the much smaller amplitude. The $\mathrm{OH}$ stretching frequency $\left(\nu_{39}\right)$ is shifted by $168 \mathrm{~cm}^{-1}$ to the red. The vibrational amplitudes for these three modes are depicted in Fig. 2 with corresponding potential energy curves shown in Fig. 3. While the anharmonic potential of the $\mathrm{OH}$ stretching mode resembles a Morse oscillator, the potential for the $\mathrm{COH}$ bending mode is steeper than a harmonic potential in a symmetric fashion. Other modes involving notable acid group $\mathrm{H}$ atom movement are the in-plane $\mathrm{COH}$ bend $\left(\nu_{25}\right)$ with a blue shift of $14 \mathrm{~cm}^{-1}$ as well as the intramolecular torsion $\left(\nu_{1}\right)$ with a blue shift of $19 \mathrm{~cm}^{-1}$. Furthermore, significant anharmonic effects are found for modes that involve motion of the hydrogen atoms in the benzoic moiety (not included in the table): Various $\mathrm{CH}$ stretching frequencies are found to be shifted by $\pm(50 \ldots 70) \mathrm{cm}^{-1}$, while the $\mathrm{CCH}$ bending frequencies are typically shifted by $\pm(10 \ldots 20) \mathrm{cm}^{-1}$.

Dimer. The results of an analogous B3LYP/D95(d,p) vibrational analysis for the dimer are given in Tab. IV] As discussed in Sec. III A, the modes involving motions of the acid group are strongly affected by the double hydrogen bond. Both the frequency shifts with respect to the monomer values and the splittings of gerade and ungerade mode frequencies are particularly large. Our results are summarized in Tab. IV. The harmonic frequencies of the symmetric $\left(\nu_{73}, A_{g}\right)$ and antisymmetric $\left(\nu_{74}, B_{u}\right) \mathrm{OH}$ stretching modes are strongly red shifted by 836 and $712 \mathrm{~cm}^{-1}$ with respect to the monomeric vibration. The anharmonicity leads to an additional red shift of $229 \mathrm{~cm}^{-1}\left(\nu_{73}\right)$ and a blue shift of $113 \mathrm{~cm}^{-1}$ $\left(\nu_{74}\right)$ thus further increasing the difference between symmetric and anti-symmetric vibration frequencies. While the $\mathrm{OH}$ stretching frequency is discussed extensively in the literature [4, 5, 8, the $\mathrm{OH}$ bending frequency has received relatively little attention: Within the harmonic approach, the symmetric $\left(\nu_{39}, B_{g}\right)$ and antisymmetric $\left(\nu_{46}, A_{u}\right)$ out-of-plane bending motions are strongly blue shifted (409 and $456 \mathrm{~cm}^{-1}$, resp.) with respect to the monomer 
vibration. The diagonal anharmonicity further shifts these frequencies by 44 and $39 \mathrm{~cm}^{-1}$ towards higher values. Apart from these outstanding cases, the effect of anharmonicity is much weaker for the dimer than for the monomer. For example, the in-plane $\mathrm{COH}$ bending mode which is blue-shifted upon dimerization by a little more than $100 \mathrm{~cm}^{-1}$ becomes essentially harmonic. The same is true for the $\mathrm{CCH}$ bending modes $\left(\leq 6 \mathrm{~cm}^{-1}\right)$ and also the anharmonic effect on the $\mathrm{CH}$ stretching vibrations is reduced by one half.

Comparison with perturbation theory. Finally, we compare the fundamental frequencies obtained from numerically exact results of the Schrödinger equation with those obtained from perturbation theory, see Eq. (1 2). The first question of interest is how precisely the one-dimensional potential scans can be reproduced by the truncated Taylor expansion around the optimized geometry. In all cases discussed above (monomer and dimer), the quartic polynomials provide a reasonably good approximation, at least within the energy range of the fundamentally excited states, see Fig. 3 for the $\nu_{8}, \nu_{12}$, and $\nu_{39}$ mode of the benzoic acid monomer. Based on this finding, the use of second order perturbation theory can be validated. For one-dimensional calculations of the anharmonic shifts of fundamental excitation frequencies, Eqs. (1/2) yield

$$
\Delta \nu_{r}^{1 D}=2\left(\phi_{r r r r}-\frac{5}{48} \frac{\phi_{r r r}^{2}}{\omega_{r}}\right)
$$

Note that the cubic constants $\phi_{r r r}$ vanish for all modes except those of $A^{\prime}$ symmetry (monomer, $C_{s}$ ) and $A_{g}$ symmetry (dimer, $C_{2 h}$ ). The anharmonic shifts are also included in Tabs. III and IV. For the cases with small relative corrections, the numerically exact results are fairly well reproduced by perturbation theory. This includes the out-of-plane $\mathrm{COH}$ bending modes of the dimer $\left(\nu_{39}, \nu_{46}\right)$ as well as the $\mathrm{OH}$ stretching modes of both monomer $\left(\nu_{39}\right)$ and dimer $\left(\nu_{73}, \nu_{74}\right)$, where the anharmonic shifts reach up to nearly $10 \%$. However, major discrepancies are found for modes with strong anharmonic effects: Apart from the low frequency intramolecular and intermolecular torsional modes of monomer $\left(\nu_{1}\right)$ and dimer $\left(\nu_{2}\right)$, respectively, the very large blue shift of about $30 \%\left(173 \mathrm{~cm}^{-1}\right)$ of the out-of-plane $\mathrm{COH}$ bending mode of the monomer $\left(\nu_{8}\right)$ is strongly overestimated $\left(339 \mathrm{~cm}^{-1}\right)$ in standard perturbation theory. Obviously, these cases are beyond the regime of PT2 treatment. 


\section{Non-perturbative vibrational analysis of anharmonic coupling}

Based on the frequencies obtained from the one-dimensional bound state calculations, we now proceed to explore the effect of anharmonic coupling at the B3LYP/D95(d,p) level. Here we restrict ourselves to the out-of-plane bending vibration $\left(\nu_{8}\right)$ of the benzoic acid monomer and the antisymmetric (IR-active) combination $\left(\nu_{46}\right)$ for the dimer. As has been mentioned above, this mode has been found to be strongly affected by the dimerization. Moreover, it is strongly anharmonic. In order to study anharmonically coupled vibrations, we perform 2-dimensional scans of the potential energy surface spanned by pairs of normal modes and solve the stationary Schrödinger equation numerically using the propagation in imaginary time.

Monomer. Our results for the benzoic acid monomer are summarized in Tab. V], Note that only those cases are listed where the fundamental frequencies obtained in the twodimensional pictures differ by at least $3 \mathrm{~cm}^{-1}$ from the results of the corresponding onedimensional calculations. With the exception of the coupling to the umbrella mode $\left(\nu_{12}\right)$ and the $\mathrm{OH}$ stretching mode $\left(\nu_{39}\right)$, all other couplings lead to shifts of the $\mathrm{COH}$ out-of-plane bending mode $\left(\nu_{8}\right)$ by $4 \ldots 18 \mathrm{~cm}^{-1}$ towards higher frequencies. An unusual case of strong coupling arises for modes $\nu_{8}$ and $\nu_{12}$, the latter of which involves some COH out-of-plane bending, too, see Fig. 2 (b). The tilted shape of the isoenergy contours shown in Fig. 4 (a) is due to the addition or subtraction of the acid group $\mathrm{H}$ atom out-of-plane amplitude connected with the two modes. Note that this is the only case where the nodal lines of the vibrational wavefunctions are not essentially parallel to the normal coordinate axes thereby not allowing an unambiguous identification of the vibrationally excited states to either one of the participating modes. The resulting anharmonic wavenumbers are 713 and $813 \mathrm{~cm}^{-1}$, where we choose the latter one which is due to an in-phase combination of the H atom motions and, hence, bears much higher IR absorption. The largest anharmonic coupling effect, however, is found for the $\mathrm{OH}$ stretching mode $\nu_{39}$. Within our two-dimensional picture, this coupling reduces the $\mathrm{COH}$ out-of-plane bending frequency by $200 \mathrm{~cm}^{-1}$. The reason for this peculiarity becomes obvious from Fig. 4 (ab). Both the strong diagonal anharmonicity of $\nu_{8}$ as well as the large coupling to $\nu_{39}$ is due to the definition of normal coordinates as rectilinear coordinates which do not represent the curvilinear nature of the large amplitude bending motion of the $\mathrm{H}$ atom appropriately. Finally, there are also anharmonic couplings 
to the remaining 26 modes not listed in Tab. $\mathrm{V}$ because the effect on the $\mathrm{COH}$ bending frequency is below $\pm 3 \mathrm{~cm}^{-1}$ in each of those cases. Since these shifts are in almost all cases towards higher frequencies, the resulting effect is a blue shift of $56 \mathrm{~cm}^{-1}$ if one assumes simple additivity of the pairwise couplings. The strong mixing of modes $\nu_{8}$ and $\nu_{12}$ together with the strong coupling of each of those modes to the $\mathrm{OH}$ stretching mode $\nu_{39}$ lead us to investigate the presence of possible three-mode coupling effects, too. A bound state calculation for a three-dimensional potential energy surface yields a red shift of the $\mathrm{COH}$ bending mode $\left(\nu_{8}\right)$ by $168 \mathrm{~cm}^{-1}$ which, however, does not substantially differ from the sum of the two-mode couplings to the $\nu_{12}\left(+46 \mathrm{~cm}^{-1}\right)$ and $\nu_{39}\left(-200 \mathrm{~cm}^{-1}\right)$ modes. It is noted that the same calculation shows that the umbrella mode $\left(\nu_{12}\right)$ is practically unshifted with respect to the one-dimensional calculation which is not expected on the basis of the pairwise couplings to the $\nu_{8}\left(-25 \mathrm{~cm}^{-1}\right)$ and to the $\nu_{39}\left(-13 \mathrm{~cm}^{-1}\right)$ modes. An inspection of the corresponding potential energy functions reveals that the strong coupling of $\nu_{8}$ and $\nu_{12}$ (see Fig. 4 a) is not present in the three-dimensional calculation but is rather a peculiarity for the case of vanishing displacement of the $\mathrm{OH}$ stretching mode $\left(\nu_{39}\right)$ only.

Dimer. Results of an analogous analysis of anharmonic coupling effects on the antisymmetric $\mathrm{COH}$ out-of-plane bending mode $\left(\nu_{46}\right)$ of the benzoic acid dimer are collected in Tab. VI. Again, only modes are included where the coupling shifts the frequencies by at least $3 \mathrm{~cm}^{-1}$. Because of the reduced vibrational amplitudes of the atoms participating in the double hydrogen bond, there are much fewer and weaker anharmonic couplings for the dimer than for the monomer. Among the few cases of relatively strong coupling, there is a significant Davydov-type coupling with the symmetric $\mathrm{COH}$ bending mode $\left(\nu_{39}\right)$. The corresponding isoenergy contours are slightly diamonds-shaped, see Fig. 5(a). This leads to an increase (blue shift) of the fundamental frequency of the antisymmetric mode $\left(\nu_{46}\right)$ by 31 $\mathrm{cm}^{-1}$. Moreover, there are strong couplings to the symmetric $\left(\nu_{73}\right)$ and antisymmetric $\left(\nu_{74}\right)$ $\mathrm{OH}$ stretching modes. Within our two-dimensional calculations these interactions give rise to red shifts of the $\nu_{46}$ frequency by 43 or $54 \mathrm{~cm}^{-1}$, respectively. The corresponding potential energy surfaces are shown in Fig. 5 (b,c). Although the potential surface spanned by the $\nu_{46}$ and $\nu_{73}$ normal coordinate is qualitatively similar to that for the monomer as depicted in Fig. 4 (b), the amplitude of the $\mathrm{COH}$ bending is much reduced thus rendering its curvilinear nature less important. Due to the different symmetry, the potential energy surface spanned by $\nu_{46}$ and $\nu_{74}$ normal coordinates is qualitatively different with slightly rectangular-shaped 
contours. In addition, there are notable couplings to two other intramolecular modes $\left(\nu_{6}\right.$, $\left.\nu_{58}\right)$ as well as to the intermolecular stretching mode $\left(\nu_{8}\right)$ each of which does not exceed $10 \mathrm{~cm}^{-1}$. Finally, the anharmonic couplings to the remaining 66 modes not listed here are again in almost all cases towards higher frequencies, so that the resulting effect is a blue shift of $72 \mathrm{~cm}^{-1}$ assuming simple additivity.

Following a similar argument as for the case of the coupling of the $\nu_{8}, \nu_{12}$, and $\nu_{39}$ modes of the monomer, the strong coupling between the antisymmetric $\mathrm{COH}$ bending mode $\nu_{46}$ of the dimer and each of the three modes $\nu_{39}, \nu_{73}$, and $\nu_{74}$ motivates three-dimensional calculations. The anharmonic coupling to the two $\mathrm{OH}$ stretching modes results in a red shift of the $\nu_{46}$ mode by $41 \mathrm{~cm}^{-1}$ which is quite different from the sum of the above pairwise couplings to modes $\nu_{73}\left(-43 \mathrm{~cm}^{-1}\right)$ and $\nu_{74}\left(-54 \mathrm{~cm}^{-1}\right)$. Also the three-mode-coupling to modes $\nu_{39}$ and $\nu_{73}$ yielding $-16 \mathrm{~cm}^{-1}$ and to modes $\nu_{39}$ and $\nu_{74}$ yielding $-42 \mathrm{~cm}^{-1}$ differ substantially from the pairwise picture.

Comparison with perturbation theory. As in the case of the one-dimensional calculations discussed above, we want to compare our results with those obtained from the standard perturbation approach. First of all, the polynomial expansion underlying Eq. (2) is reviewed. While in almost all cases the two-dimensional scans of the potential energy surface are reproduced very well, there are a few remarkable exceptions for the above mentioned cases of strong coupling: The curved shape of the potential well spanned by the $\mathrm{COH}$ bending $\left(\nu_{8}\right)$ and $\mathrm{OH}$ stretching $\left(\nu_{39}\right)$ mode of the monomer is not even qualitatively approximated by a quartic expansion, see dashed contours in Fig. 4 (b). Although to a lesser extent, the same is true for the potential function spanned by $\nu_{46}, \nu_{73}$ of the dimer, see Fig. 5 (b). Also the tilted shape of the contours of the potential surface spanned by the $\mathrm{COH}$ bending $\left(\nu_{8}\right)$ and the umbrella $\left(\nu_{12}\right)$ normal coordinates of the monomer (Fig. 4 a) cannot be reproduced by diagonal and semi-diagonal quartic terms only. However, inclusion of the (rather large) force constant $\phi_{8,8,8,12}$ would lead to very good agreement. In contrast, the dimer potentials in the $\left(\nu_{39}, \nu_{46}\right)$ and $\left(\nu_{46}, \nu_{74}\right)$ planes are well reproduced by (relatively large) positive and negative semi-diagonal quartic force constants.

Next, we compare our numerically exact solutions of the time-independent Schrödinger equation with those obtained from perturbation theory of Eqs. (1) 2). The shift of the 
fundamental excitation frequencies $\nu_{r}$ due to anharmonic coupling to mode $\nu_{s}$ is given by

$$
\Delta \nu_{r}^{2 D}=\frac{1}{8}\left(\phi_{r r s s}-\frac{\phi_{r r r} \phi_{r s s}}{\omega_{r}}-\frac{\phi_{r r s}}{\omega_{s}}\left[\phi_{s s s}+\phi_{r r s} \frac{8 \omega_{r}^{2}-3 \omega_{s}^{2}}{4 \omega_{r}^{2}-\omega_{s}^{2}}\right]\right)
$$

These values are included for comparison in Tabs. V and VI. In addition to those cases where the truncated Taylor expansion fails (e. g. coupling of the $\nu_{8}$ mode to $\nu_{12}$ and $\nu_{39}$ of the monomer, ...) also most of the other anharmonic couplings are not reproduced correctly by perturbation theory, apparently regardless of the relative size of the shifts. The failure of the second order approach is much more pronounced than in the case of the one-dimensional data compiled in Tabs III and IV where large deviations are limited to the cases of unusually strong anharmonic effects.

\section{CONCLUSIONS}

An anharmonic vibrational analysis of benzoic acid monomer and dimer has been carried out at the DFT/B3LYP level of theory employing D95(d,p) and cc-pVTZ basis sets. The standard second order perturbation (PT2) approach yields very good agreement with measured absorption spectra in the mid-infrared regime $\left(500 \ldots 1800 \mathrm{~cm}^{-1}\right)$ [6]. The frequencies rarely deviate by more than $1 \%$, in most cases even considerably less. Although these results are certainly better than expected, they may be to some extent caused by error cancelations in the computational approach, at least for the spectral region considered here. The infrared intensities are reproduced qualitatively where the absorption patterns are notably better for the larger of the two basis sets (cc-pVTZ). The total effect of anharmonic coupling leads to red shifts of the fundamental excitation frequencies by about $1 \ldots 3 \%$. This is approximately in accord with the common practice of empirically scaling harmonic frequencies in order to match experimental spectra. Of particular interest is the out-of-plane $\mathrm{COH}$ bending mode of the monomer $\left(\nu_{8}\right)$ which exhibits an extreme blue shift upon dimerization $\left(\nu_{39}, \nu_{46}\right)$ which has motivated the present non-perturbative studies of anharmonic effects in reduced dimensionality. The results of our one-, two-, and three-

dimensional studies show two peculiarities: (1) The relatively small total anharmonic shift of the monomer $\mathrm{COH}$ bending frequency $\left(\nu_{8}\right)$ of $20 \mathrm{~cm}^{-1}$ comprise of many individual shifts which are of the order of a few hundreds of $\mathrm{cm}^{-1}$ and which are canceling each other for the most part. Although the total anharmonic effects for the dimer are in general much less 
pronounced, it is again found that - due to similar cancelation effects as for the monomer the total anharmonic frequency shift of $13 \mathrm{~cm}$ for the $\nu_{46}$ mode is much smaller than some of its constituents. (2) Within the low-dimensional pictures it has been shown that the conditions for the standard PT2 perturbative approach are not always fulfilled. In some cases, the underlying truncation of the Taylor series containing only cubic and semi-diagonal quartic force constants is inappropriate, in other cases the second order treatment is insufficient.

Given these findings, the results of anharmonic calculations for the $\mathrm{COH}$ out-of-plane vibrational mode have to be considered critically: The strong diagonal anharmonic shift of the $\nu_{8}$ mode $\left(+173 \mathrm{~cm}^{-1}\right)$ of the monomer is almost compensated by the (combined) anharmonic coupling $\left(-168 \mathrm{~cm}^{-1}\right)$ to the umbrella mode $\nu_{12}$ and the $\mathrm{OH}$ stretching vibration $\nu_{39}$ thus suggesting a rather small anharmonic effect. However, the nearly perfect match of the theoretical (PT2) values of $574 \mathrm{~cm}^{-1}$ (D95(d,p)) or $575 \mathrm{~cm}^{-1}$ (cc-pVTZ) and the experimental value of $571 \mathrm{~cm}^{-1}$ could be partially accidental. The antisymmetric mode $\mathrm{COH}$ bending mode $\left(\nu_{46}\right)$ of the dimer is the only case where our harmonic results obtained for the two basis sets deviate by more than $1 \%$ from each other. Moreover, the $8 \%$ or $3 \%$ difference between anharmonic (PT2) values of $1037 \mathrm{~cm}^{-1}$ (D95(d,p)) or 992/995 cm-1 (ccpVTZ) and the experimental value of $962 \mathrm{~cm}^{-1}$ represents the largest discrepancy within the mid-IR regime considered here. At present, this cannot be explained satisfactorily by our non-perturbative results either: We have identified strong anharmonic coupling to three modes (symmetric $\mathrm{COH}$ bending $\nu_{39}$ as well as the two $\mathrm{OH}$ stretching modes $\nu_{73}, \nu_{74}$ ). The shifts obtained from non-perturbative calculations of anharmonic coupling to any pair of these modes deviate substantially from the sum of the respective pairwise calculations. Hence, it is anticipated that (at least) four-dimensional calculations are required to finally clarify the question of the anharmonic effect on the $\nu_{46}$ vibrational mode of the benzoic acid dimer. Moreover, in order to ensure convergence of the electronic structure calculations for this particular vibrational frequency, even larger basis sets and/or higher quantum chemical methods may be required. However, either of these approaches are currently beyond the available computational means. 


\section{Acknowledgments}

Financial support by the Deutsche Forschungsgemeinschaft through program SFB 450 on "Analysis and control of ultrafast photoinduced reactions" is gratefully acknowledged. The quantum-chemical calculations were carried out on an IBM pSeries 690 supercomputer available at the HLRN. We thank Dr. Bernd Kallies for valuable help concerning the quantum-chemical calculations.

[1] F. Madeja and M. Havenith, J. Chem. Phys. 117, 7162 (2002), URL http://dx.doi.org/ $10.1063 / 1.1507581$

[2] P. R. L. Markwick, N. L. Doltsinis, and D. Marx, J. Chem. Phys. 122, 054112 (2005), URL http://dx.doi.org/10.1063/1.1842049

[3] Z. Smedarchina, A. Fernandez-Ramos, and W. Siebrand, J. Chem. Phys. 122, 134309 (2005), URL http://dx.doi .org/10.1063/1.1868552.

[4] G. M. Florio, E. L. Sibert III, and T. S. Zwier, Faraday Discuss. 118, 315 (2001), URL http://dx.doi.org/10.1039/b009594j.

[5] G. M. Florio, T. S. Zwier, E. M. Myshakin, K. D. Jordan, and E. L. Sibert III, J. Chem. Phys. 118, 1735 (2003), URL http://dx.doi.org/10.1063/1.1530573.

[6] J. M. Bakker, L. M. Aleese, G. von Helden, and G. Meijer, J. Chem. Phys. 119, 11180 (2003), URL http://dx.doi .org/10.1063/1.1622657.

[7] M. V. Vener, O. Kühn, and J. M. Bowman, Chem. Phys. Lett. 349, 562 (2001), URL http: //dx.doi.org/10.1016/S0009-2614(01)01248-9.

[8] E. M. Myshakin, K. D. Jordan, E. L. Sibert III, and M. A. Johnson, J. Chem. Phys. 119, 10138 (2003), URL http://dx.doi.org/10.1063/1.1616918.

[9] M. V. Vener, O. Kühn, and J. Sauer, J. Chem. Phys. 114, 240 (2001), URL http://dx.doi. org/10.1063/1.1330748.

[10] K. R. Asmis, N. L. Pivonka, G. Santambrogio, M. Brümmer, C. Kaposta, D. M. Neumark, and L. Wöste, Science 299, 1375 (2003), URL http://dx.doi.org/10.1126/science.1081634.

[11] D. T. Moore, J. Oomens, L. van der Meer, G. von Helden, G. Meijer, J. Valle, A. G. Marshall, and J. R. Eyler, ChemPhysChem 5, 740 (2004), URL http://dx.doi.org/10.1002/cphc. 


\section{2}

[12] J. M. Bakker, I. Compagnon, G. Meijer, G. von Helden, M. Kabelac, P. Hobza, and M. S. de Vries, Phys. Chem. Chem. Phys. 6, 2810 (2004), URL http://dx.doi.org/10.1039/ b316158g.

[13] S. G. Stepanian, I. D. Reva, E. D. Radchenko, and G. G. Sheina, Vib. Spec. 11, 123 (1996), URL http://dx.doi.org/10.1016/0924-2031(95)00068-2.

[14] E. G. Robertson and J. P. Simons, Phys. Chem. Chem. Phys. 3, 1 (2001), URL http://dx. doi.org/10.1039/b008225m.

[15] M. A. Palafox, J. L. Nunez, and M. Gil, Int. J. Quant. Chem. 89, 1 (2002), URL http: //dx.doi.org/10.1002/qua.10202.

[16] K. Heyne, N. Huse, J. Dreyer, E. T. J. Nibbering, T. Elsaesser, and S. Mukamel, J. Chem. Phys. 121, 902 (2004), URL http://dx.doi.org/10.1063/1.1762873.

[17] J. C. Light and T. Carrington, Adv. Chem. Phys. 114, 263 (2000).

[18] H. H. Nielsen, in Handbuch der Physik, edited by S. Flügge (Springer, Berlin, 1959), vol. 37, p. 173.

[19] I. A. Mills, in Molecular Spectroscopy: Modern Research, edited by K. N. Rao and C. W. Mathews (Academic Press, New York, 1972), chap. 3, p. 115.

[20] D. Papouŝek and M. R. Aliev, Molecular Vibrational-Rotational Spectra (Elsevier, Amsterdam, 1982).

[21] V. Barone, J. Chem. Phys. 120, 3059 (2004), URL http://dx.doi.org/10.1063/1.1637580.

[22] V. Barone, J. Chem. Phys. 122, 014108 (2005), URL http://dx.doi.org/10.1063/1. 1824881 .

[23] G. M. Chaban, J. O. Jung, and R. B. Gerber, J. Chem. Phys. 111, 1823 (1999), URL http: //dx.doi.org/10.1063/1.479452.

[24] L. S. Norris, M. A. Ratner, A. E. Roitberg, and R. B. Gerber, J. Chem. Phys. 105, 11261 (1996), URL http://dx.doi.org/10.1063/1.472922.

[25] O. Christiansen, J. Chem. Phys. 119, 5773 (2003), URL http://dx.doi.org/10.1063/1. 1601593 .

[26] O. Christiansen, J. Chem. Phys. 120, 2149 (2004), URL http://dx.doi.org/10.1063/1. 1637579 .

[27] A. D. Becke, J. Comp. Chem. 20, 63 (1999), URL http://dx.doi.org/10.1002/(SICI) 
1096-987X (19990115) 20:1<63: :AID-JCC8>3.0.C0;2-A.

[28] M. J. Frisch, G. W. Trucks, H. B. Schlegel, G. E. Scuseria, M. A. Robb, J. R. Cheeseman, J. A. Montgomery, Jr., T. Vreven, K. N. Kudin, J. C. Burant, et al., Gaussian 03, Revision B.05, Gaussian, Inc., Pittsburgh PA (2003), URL http://www.gaussian.com.

[29] T. H. Dunning Jr., J. Chem. Phys. 53, 2823 (1970), URL http://dx.doi.org/10.1063/1. 1674408 .

[30] T. H. Dunning Jr., J. Chem. Phys. 90, 1007 (1989), URL http://dx.doi.org/10.1063/1. 456153 .

[31] W. Schneider and W. Thiel, Chem. Phys. Lett. 157, 367 (1989), URL http://dx.doi.org/ 10.1016/0009-2614(89)87263-X.

[32] M. Cohen and S. Kais, J. Phys. A 19, 683 (1986), URL http://dx.doi.org/10.1088/ 0305-4470/19/5/021.

[33] M. Skala, J. Cizek, and J. Zamastil, J. Phys. A 32, 5715 (1999), URL http://dx.doi.org/ $10.1088 / 0305-4470 / 32 / 30 / 314$.

[34] R. Kosloff and H. Tal-Ezer, Chem. Phys. Lett. 127, 223 (1986), URL http://dx.doi.org/ 10.1016/0009-2614(86)80262-7.

[35] R. Kosloff, Annu. Rev. Phys. Chem. 45, 145 (1994), URL http://dx.doi.org/10.1146/ annurev.pc.45.100194.001045.

[36] Complete tables of harmonic and anharmonic force constants and frequencies are available from the author's web site, URL http://page.mi.fu-berlin.de/〜burkhard/PhysChem/ ClusterVibSpec. 
TABLE I: Theoretical (B3LYP) infrared intensities (in $\mathrm{km} / \mathrm{mol}$ ), harmonic and anharmonic frequencies (in $\mathrm{cm}^{-1}$ ) for two different basis sets (D95(d,p) and cc-pVTZ) versus observed frequencies [6] for vibrational normal modes of the benzoic acid monomer $\left(C_{s}\right)$. In both cases, anharmonic corrections are based on second order perturbation, see Eq. (1), for the smaller of the two basis sets $(\mathrm{D} 95(\mathrm{~d}, \mathrm{p}))$

\begin{tabular}{|c|c|c|c|c|c|c|c|c|c|}
\hline \multicolumn{2}{|c|}{ Mode } & \multicolumn{3}{|c|}{$\mathrm{D} 95(\mathrm{~d}, \mathrm{p})$} & \multicolumn{3}{|c|}{ cc-pVTZ } & \multirow{3}{*}{$\begin{array}{l}\text { Obs. } \\
571\end{array}$} & \multirow{3}{*}{$\begin{array}{l}\text { Description } \\
\mathrm{C}-\mathrm{O}-\mathrm{H} \mathrm{b}\end{array}$} \\
\hline & & \multirow{2}{*}{$\begin{array}{c}\text { Int. } \\
71\end{array}$} & \multirow{2}{*}{$\begin{array}{c}\text { Harm. } \\
594\end{array}$} & \multirow{2}{*}{$\begin{array}{c}\text { Anh. } \\
574\end{array}$} & \multirow{2}{*}{$\begin{array}{c}\text { Int. } \\
61\end{array}$} & \multirow{2}{*}{$\begin{array}{c}\text { Harm. } \\
595\end{array}$} & \multirow{2}{*}{$\begin{array}{c}\text { Anh. } \\
575\end{array}$} & & \\
\hline 8 & A" & & & & & & & & \\
\hline 10 & $A^{\prime}$ & 50 & 630 & 623 & 49 & 640 & 633 & 631 & rg C-C-C b \\
\hline 11 & A" & 6 & 700 & 685 & 10 & 710 & 695 & 688 & rg C-C-H b \\
\hline 12 & A" & 151 & 726 & 710 & 118 & 732 & 716 & 710 & rg C-C-H b (u) \\
\hline 13 & $A^{\prime}$ & 9 & 771 & 761 & 9 & 777 & 767 & 767 & $\mathrm{C}-\mathrm{O}-\mathrm{H} \mathrm{b}, \mathrm{rg}$ def \\
\hline 20 & $A^{\prime}$ & 15 & 1039 & 1025 & 17 & 1050 & 1036 & 1026 & rg C-C-H b \\
\hline 21 & $A^{\prime}$ & 64 & 1090 & 1068 & 107 & 1094 & 1072 & 1063 & rg C-C-H b \\
\hline 22 & $A^{\prime}$ & 58 & 1117 & 1099 & 43 & 1118 & 1100 & 1084 & rg C-C-H b \\
\hline 24 & $A^{\prime}$ & 95 & 1186 & 1172 & 159 & 1191 & 1177 & 1173 & $\mathrm{C}-\mathrm{C}-\mathrm{H} \mathrm{b}, \mathrm{C}-\mathrm{O}-\mathrm{H} \mathrm{b}$ \\
\hline 25 & $A^{\prime}$ & 148 & 1208 & 1180 & 62 & 1212 & 1184 & 1187 & C-C-H b, C-O-H b \\
\hline 28 & $A^{\prime}$ & 130 & 1381 & 1343 & 113 & 1372 & 1334 & 1347 & $\mathrm{C}-\mathrm{C}-\mathrm{H}$ b, C-O-H b \\
\hline 29 & $A^{\prime}$ & 18 & 1474 & 1452 & 16 & 1488 & 1466 & 1455 & $\mathrm{C}-\mathrm{C} \mathrm{s}, \mathrm{rg}$ def \\
\hline 31 & $A^{\prime}$ & 5 & 1628 & 1592 & 5 & 1625 & 1589 & 1591 & $\mathrm{C}-\mathrm{C} \mathrm{s}, \mathrm{rg}$ def \\
\hline 32 & $A^{\prime}$ & 18 & 1649 & 1612 & 17 & 1646 & 1609 & 1609 & C-C s, rg def \\
\hline 33 & $A^{\prime}$ & 348 & 1804 & 1770 & 331 & 1792 & 1758 & 1752 & $\mathrm{C}=\mathrm{O} \mathrm{s}, \mathrm{C}-\mathrm{O}-\mathrm{H} \mathrm{b}$ \\
\hline
\end{tabular}


TABLE II: Same as Tab. I but for the cyclic benzoic acid dimer $\left(C_{2 h}\right)$. Bracketed numbers in the last column indicate the index of the corresponding monomeric mode of vibration.

\begin{tabular}{|c|c|c|c|c|c|c|c|c|c|}
\hline \multicolumn{2}{|c|}{ Mode } & \multicolumn{3}{|c|}{$\mathrm{D} 95(\mathrm{~d}, \mathrm{p})$} & \multicolumn{3}{|c|}{ cc-pVTZ } & \multirow{3}{*}{$\begin{array}{l}\text { Obs. } \\
540\end{array}$} & \multirow{3}{*}{$\begin{array}{l}\text { Description } \\
\text { acid groups rocking }(7)\end{array}$} \\
\hline & & \multirow{2}{*}{$\begin{array}{l}\text { Int. } \\
131\end{array}$} & \multirow{2}{*}{$\begin{array}{c}\text { Harm. } \\
554\end{array}$} & \multirow{2}{*}{$\begin{array}{c}\text { Anh. } \\
555\end{array}$} & \multirow{2}{*}{$\begin{array}{l}\text { Int. } \\
114\end{array}$} & \multirow{2}{*}{$\begin{array}{c}\text { Harm. } \\
553\end{array}$} & \multirow{2}{*}{$\begin{array}{c}\text { Anh. } \\
554\end{array}$} & & \\
\hline 20 & $B_{u}$ & & & & & & & & \\
\hline 24 & $B_{u}$ & 44 & 673 & 668 & 45 & 683 & 678 & 664 & acid groups scissor (10) \\
\hline 25 & $A_{u}$ & 15 & 695 & 683 & 20 & 706 & 694 & 682 & rg C-C-H b (11) \\
\hline 27 & $A_{u}$ & 163 & 720 & 711 & 133 & 728 & 719 & 708 & rg C-C-H b (u) (12) \\
\hline 30 & $B_{u}$ & 22 & 815 & 808 & 25 & 822 & 815 & 797 & acid groups scissor, rg def (13) \\
\hline 32 & $A_{u}$ & 17 & 820 & 806 & 8 & 831 & 817 & 810 & rg C-C-H b (14) \\
\hline 44 & $B_{u}$ & 24 & 1040 & 1023 & 22 & 1050 & 1033 & 1022 & rg C-C-H b (20) \\
\hline 46 & $A_{u}$ & 222 & 1050 & 1037 & $\begin{array}{c}104 \\
55\end{array}$ & $\begin{array}{l}1005 \\
1008\end{array}$ & $\begin{array}{l}992 \\
995\end{array}$ & 962 & rg C-C-H b, C-O-H b (8) \\
\hline 48 & $B_{u}$ & 24 & 1096 & 1068 & 20 & 1104 & 1076 & 1066 & rg C-C-H b (21) \\
\hline 49 & $B_{u}$ & 8 & 1150 & 1128 & 13 & 1152 & 1130 & 1126 & rg C-C-H b (22) \\
\hline 53 & $B_{u}$ & 48 & 1191 & 1180 & 59 & 1202 & 1191 & 1176 & rg C-C-H b (24) \\
\hline 56 & $B_{u}$ & 138 & 1328 & 1304 & 556 & 1324 & 1300 & 1297 & C-C-H b, C-O-H b $(25 / 26)$ \\
\hline 58 & $B_{u}$ & 682 & 1353 & 1324 & 276 & 1349 & 1320 & 1322 & C-C-H b, C-O-H b $(25 / 26)$ \\
\hline 61 & $B_{u}$ & 76 & 1471 & 1436 & 155 & 1467 & 1432 & 1432 & C-C-H b, C-O-H b (28) \\
\hline 63 & $B_{u}$ & 201 & 1477 & 1444 & 69 & 1488 & 1455 & 1453 & C-C-H b, C-O-H b (29) \\
\hline 65 & $B_{u}$ & 51 & 1521 & 1490 & 9 & 1532 & 1501 & 1498 & C-C-H b, C-O-H b (30) \\
\hline 67 & $B_{u}$ & 52 & 1627 & 1588 & 50 & 1624 & 1585 & 1591 & C-C s, rg def (31) \\
\hline 70 & $B_{u}$ & 60 & 1649 & 1608 & 53 & 1646 & 1605 & 1618 & C-C s, rg def (32) \\
\hline 72 & $B_{u}$ & 956 & 1745 & 1704 & 900 & 1733 & 1692 & 1709 & $\mathrm{C}=\mathrm{O}$ s stretch, $\mathrm{C}-\mathrm{O}-\mathrm{H}$ b (33) \\
\hline
\end{tabular}


TABLE III: One-dimensional vibrational analysis of benzoic acid monomer $\left(C_{s}\right)$. Harmonic and anharmonic frequencies (in $\mathrm{cm}^{-1}$ ) are calculated using B3LYP/D95(d,p) density functional method (anharmonic frequency shifts in brackets). Results from corresponding one-dimensional perturbation calculations (3) are included for comparison $\left(\Delta \nu_{r}^{1 D}\right)$.

\begin{tabular}{|c|c|c|c|c|c|c|}
\hline \multicolumn{2}{|c|}{ Mode } & \multirow{2}{*}{$\begin{array}{c}\text { Harm. } \\
67\end{array}$} & \multicolumn{2}{|c|}{ Anharm. } & \multirow{2}{*}{$\begin{array}{r}\Delta \nu_{r}^{1 D} \\
+31\end{array}$} & \multirow{2}{*}{$\begin{array}{l}\text { Description } \\
\text { C-C-C-OOH t }\end{array}$} \\
\hline 1 & A" & & 86 & $(+19)$ & & \\
\hline 8 & A" & 594 & 767 & $(+173)$ & +339 & $\mathrm{C}-\mathrm{O}-\mathrm{H}$ b \\
\hline 12 & A" & 726 & 738 & $(+12)$ & +14 & rg C-C-H b (u) \\
\hline 25 & $\mathrm{~A}^{\prime}$ & 1208 & 1222 & $(+14)$ & +15 & rg C-C-H b, C-O-H b \\
\hline 39 & $\mathrm{~A}^{\prime}$ & 3785 & 3617 & $(-168)$ & -183 & $\mathrm{O}-\mathrm{H} \mathrm{s}$ \\
\hline
\end{tabular}

$\mathrm{rg}=$ ring, $\mathrm{b}=$ bend, $\mathrm{s}=$ stretch, $\mathrm{t}=$ torsion, $\mathrm{u}=$ umbrella 
TABLE IV: Same as Tab. III but for the cyclic benzoic acid dimer $\left(C_{2 h}\right)$

\begin{tabular}{|c|c|c|c|c|c|c|}
\hline \multicolumn{2}{|c|}{ Mode } & \multirow{2}{*}{$\frac{\text { Harm. }}{32}$} & \multicolumn{2}{|c|}{ Anharm. } & \multirow{2}{*}{$\frac{\Delta \nu_{r}^{1 D}}{+65}$} & \multirow{2}{*}{$\frac{\text { Description }}{\text { inter-mol. twist }}$} \\
\hline 2 & $\mathrm{~A}_{u}$ & & 55 & $(+23)$ & & \\
\hline 39 & $\mathrm{~B}_{g}$ & 1003 & 1047 & $(+44)$ & +53 & $\mathrm{C}-\mathrm{O}-\mathrm{H}$ b \\
\hline 46 & $\mathrm{~A}_{u}$ & 1050 & 1089 & $(+39)$ & +48 & C-O-H b \\
\hline 73 & $\mathrm{~A}_{g}$ & 2949 & 2720 & $(-229)$ & -210 & $\mathrm{O}-\mathrm{H} \mathrm{s}$ \\
\hline 74 & $\mathrm{~B}_{u}$ & 3073 & 3186 & $(+113)$ & +123 & $\mathrm{O}-\mathrm{H} \mathrm{s}$ \\
\hline
\end{tabular}


TABLE V: Anharmonic coupling in the benzoic acid monomer $\left(C_{s}\right)$ at B3LYP/D95(d,p) level of theory. Results of two- and three-dimensional calculations for interaction of modes $\nu_{s}$ with $\mathrm{COH}$ out-of-plane bending mode $\left(\nu_{8}, \mathrm{~A}\right.$ "). Shifts with respect to the one-dimensional anharmonic result $\left(767 \mathrm{~cm}^{-1}\right)$ are given in brackets. Results from corresponding two-dimensional perturbation calculations (4) are included for comparison $\left(\Delta \nu_{8}^{2 D}\right)$. Only modes with a coupling of at least 3 $\mathrm{cm}^{-1}$ are included.

\begin{tabular}{|c|c|c|c|c|c|c|}
\hline \multicolumn{2}{|c|}{ Mode } & \multirow{2}{*}{$\frac{\nu_{s}}{173}$} & \multicolumn{2}{|c|}{$\nu_{8}$} & \multirow{2}{*}{$\frac{\Delta \nu_{8}^{2 D}}{+23}$} & \multirow{2}{*}{$\begin{array}{l}\text { Description } \\
\mathrm{C}-\mathrm{C}-\mathrm{C}(\mathrm{OOH}) \mathrm{b}\end{array}$} \\
\hline 2 & A" & & 774 & $(+7)$ & & \\
\hline 3 & $A^{\prime}$ & 219 & 771 & $(+4)$ & +4 & $\mathrm{C}-\mathrm{C}-\mathrm{C}(\mathrm{OOH}) \mathrm{b}$ \\
\hline 6 & A" & 444 & 785 & $(+18)$ & +37 & rg C-C-C b, C-O-H b \\
\hline 7 & $A^{\prime}$ & 497 & 771 & $(+4)$ & +4 & $\mathrm{C}-\mathrm{C}-\mathrm{O}(\mathrm{H}) \mathrm{b}$ \\
\hline 10 & $A^{\prime}$ & 637 & 773 & $(+6)$ & +8 & $\mathrm{O}-\mathrm{C}-\mathrm{O} \mathrm{b}, \mathrm{rg} \mathrm{d}$ \\
\hline 11 & A" & 695 & 783 & $(+16)$ & +10 & rg C-C-H b \\
\hline 12 & A" & 713 & 813 & $(+46)$ & +28 & rg C-C-H b (u) \\
\hline 13 & $A^{\prime}$ & 774 & 771 & $(+4)$ & +4 & $\mathrm{O}-\mathrm{C}-\mathrm{O} \mathrm{b}, \mathrm{C}-\mathrm{C}(\mathrm{OOH}) \mathrm{s}, \mathrm{rg} \mathrm{d}$ \\
\hline 14 & A" & 831 & 764 & $(-3)$ & +4 & rg C-C-H, C-C-C(OOH) b \\
\hline 24 & $A^{\prime}$ & 1197 & 773 & $(+6)$ & +25 & rg C-C-H b, C-O-H b \\
\hline 25 & $A^{\prime}$ & 1235 & 780 & $(+13)$ & +17 & rg C-C-H b, C-O-H b \\
\hline 28 & $A^{\prime}$ & 1384 & 771 & $(+4)$ & +2 & $\mathrm{C}-\mathrm{O}(\mathrm{H}) \mathrm{s}, \mathrm{C}-\mathrm{O}-\mathrm{H} \mathrm{b}$ \\
\hline \multirow[t]{2}{*}{39} & $A^{\prime}$ & 3602 & 567 & $(-200)$ & -398 & $\mathrm{O}-\mathrm{H} \mathrm{s}$ \\
\hline & & & & $(+56)$ & & all other modes (sum) \\
\hline $12 / 39$ & A"/A' & $738 / 3656$ & 599 & $(-168)$ & & \\
\hline
\end{tabular}

$\mathrm{rg}=$ ring, $\mathrm{b}=$ bend, $\mathrm{s}=$ stretch, $\mathrm{d}=$ deformation, $\mathrm{u}=$ umbrella 
TABLE VI: Same as Tab. V but for the antisymmetric COH out-of-plane bending mode $\left(\nu_{46}\right.$, $\left.\mathrm{A}_{u}\right)$ of the cyclic benzoic acid dimer $\left(C_{2 h}\right)$. Shifts with respect to the one-dimensional anharmonic result $\left(1089 \mathrm{~cm}^{-1}\right)$ are given in brackets.

\begin{tabular}{|c|c|c|c|c|c|c|}
\hline \multicolumn{2}{|c|}{ Mode } & \multirow{2}{*}{$\frac{\nu_{s}}{108}$} & \multicolumn{2}{|c|}{$\nu_{46}$} & \multirow{2}{*}{$\frac{\Delta \nu_{46}^{2 D}}{+8}$} & \multirow{2}{*}{$\begin{array}{l}\text { Description } \\
\text { C-C-C-OOH t }\end{array}$} \\
\hline 6 & $\mathrm{~A}_{u}$ & & 1093 & $(+4)$ & & \\
\hline 8 & $\mathrm{~A}_{g}$ & 119 & 1081 & $(-8)$ & -10 & inter-molecular stretch \\
\hline 16 & $\mathrm{~A}_{g}$ & 442 & 1086 & $(-3)$ & -2 & $\mathrm{rg}$ C-C-C b, C-C $(\mathrm{OOH}) \mathrm{s}$ \\
\hline 39 & $\mathrm{~B}_{g}$ & 1077 & 1120 & $(+31)$ & +51 & $\mathrm{C}-\mathrm{O}-\mathrm{H} \mathrm{b}$ \\
\hline 58 & $\mathrm{~B}_{u}$ & 1356 & 1094 & $(+5)$ & +4 & rg C-C-H b, C-O-H b \\
\hline 73 & $\mathrm{~A}_{g}$ & 2551 & 1048 & $(-43)$ & -79 & $\mathrm{O}-\mathrm{H} \mathrm{s}$ \\
\hline \multirow[t]{2}{*}{74} & $\mathrm{~B}_{u}$ & 2675 & 1048 & $(-54)$ & -61 & $\mathrm{O}-\mathrm{H} \mathrm{s}$ \\
\hline & & & & $(+72)$ & & all other modes (sum) \\
\hline $73 / 74$ & $\mathrm{~A}_{g} / \mathrm{B}_{u}$ & $2551 / 2675$ & 1048 & $(-41)$ & & \\
\hline $39 / 73$ & $\mathrm{~B}_{g} / \mathrm{A}_{g}$ & $1029 / 2785$ & 1073 & $(-16)$ & & \\
\hline $39 / 74$ & $\mathrm{~B}_{g} / \mathrm{B}_{u}$ & $1004 / 3057$ & 1047 & $(-42)$ & & \\
\hline
\end{tabular}


FIG. 1: Experimental vs. calculated mid-IR spectra of benzoic acid at various levels of theory. Computed lines are convoluted with Gaussians of $1 \%$ FWHM to match the resolution of the measurements [6]. (a) Monomer. (b) Dimer

FIG. 2: (Color online) Displacement vectors for selected normal modes of the benzoic acid monomer. (a) $\mathrm{COH}$ out-of-plane bending vibration $\left(\nu_{8}, A^{\prime \prime}\right)$. (b) Umbrella vibration $\left(\nu_{12}, A^{\prime \prime}\right)$. (c) $\mathrm{OH}$ stretching vibration $\left(\nu_{39}, A^{\prime}\right)$.

FIG. 3: (Color online) Potential energy curves along selected normal modes of the benzoic acid monomer $\left(\nu_{8}, \nu_{12}\right.$, and $\left.\nu_{39}\right)$ see also Fig. 2, Full curves: B3LYP/D95(d,p) results. Dashed and dotted curves: Quadratic and quartic approximation, respectively.

FIG. 4: (Color online) Two-dimensional potential energy surfaces (in $\mathrm{cm}^{-1}$ ) of benzoic acid monomer. $\mathrm{COH}$ bending $\left(\nu_{8}\right)$ vs. (a) umbrella mode $\left(\nu_{12}\right)$ and (b) $\mathrm{OH}$ stretching mode $\left(\nu_{39}\right)$. Thick contours: B3LYP/D95(d,p) results. Thin contours: Quartic approximation. The contour spacing is $1000 \mathrm{~cm}^{-1}$.

FIG. 5: (Color online) Same as Fig. 4 but for benzoic acid dimer. Antisymmetric COH bending $\left(\nu_{46}\right)$ vs (a) symmetric $\mathrm{COH}$ bending mode $\left(\nu_{39}\right)$, (b) symmetric $\mathrm{OH}$ stretching mode $\left(\nu_{73}\right)$, and (c) antisymmetric $\mathrm{OH}$ stretching mode $\left(\nu_{74}\right)$ 
Figure 1a, Antony et al., Journal of Chemical Physics

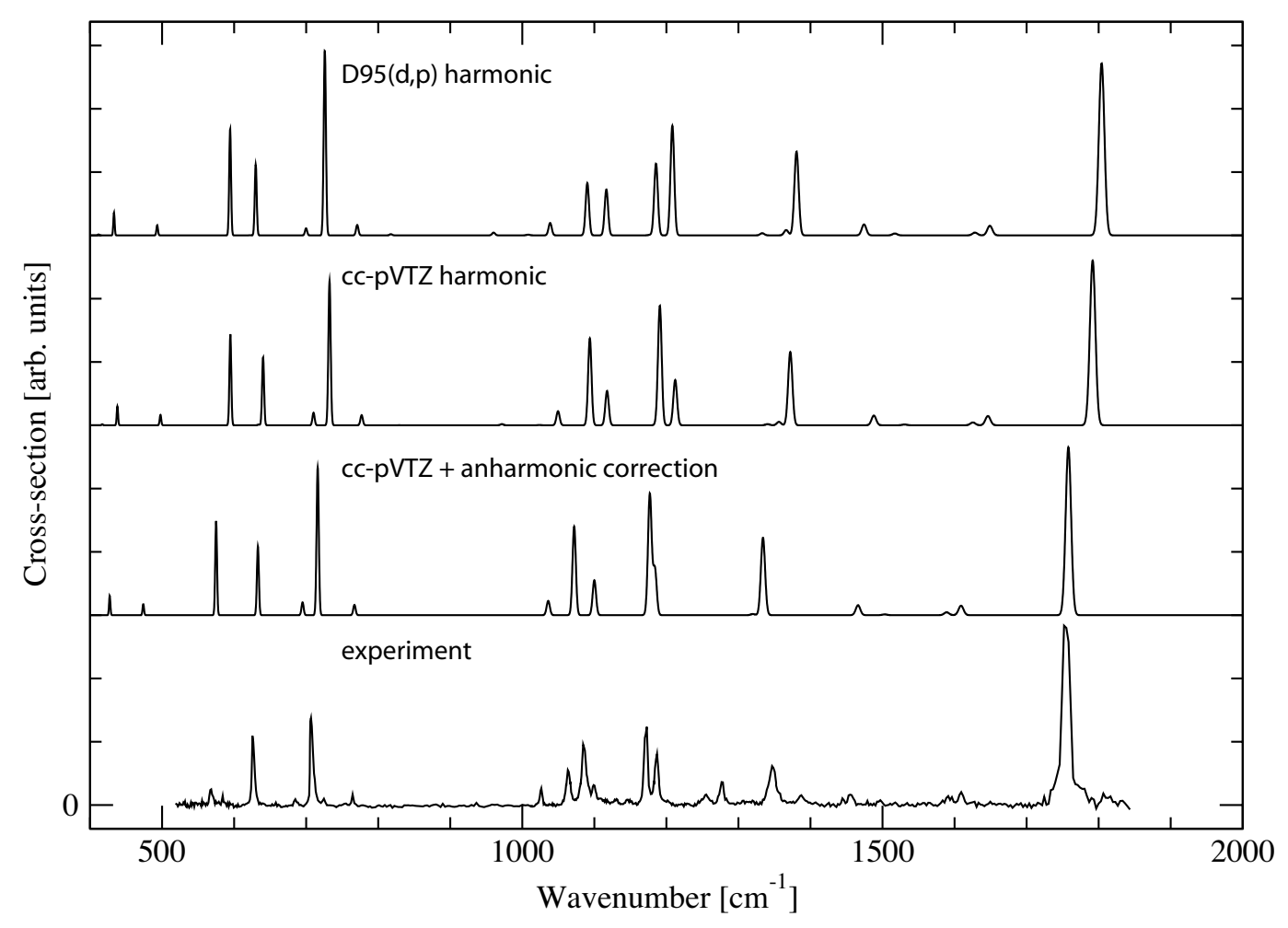


Figure 1b, Antony et al., Journal of Chemical Physics

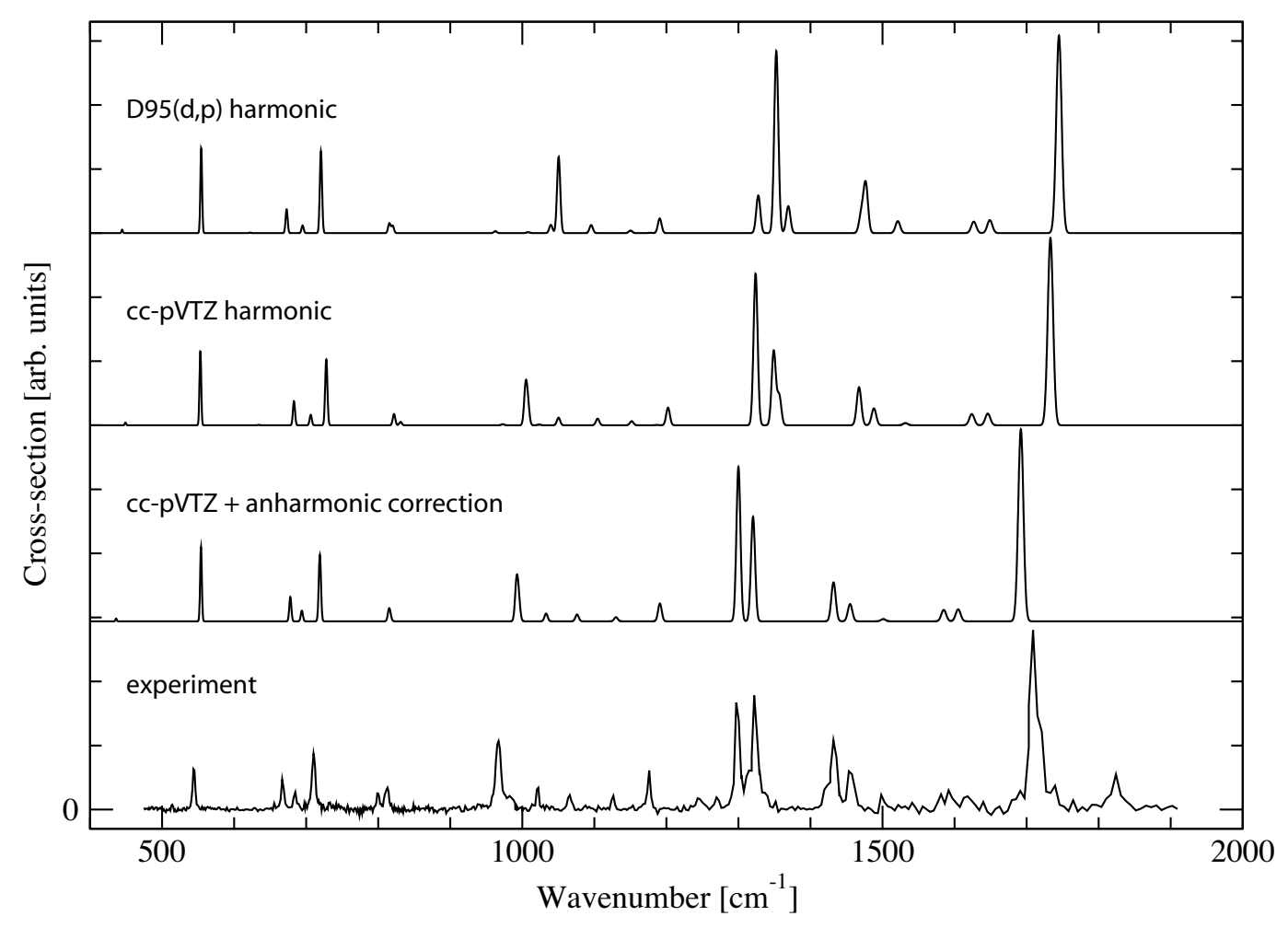


Figure 2a, Antony et al., Journal of Chemical Physics

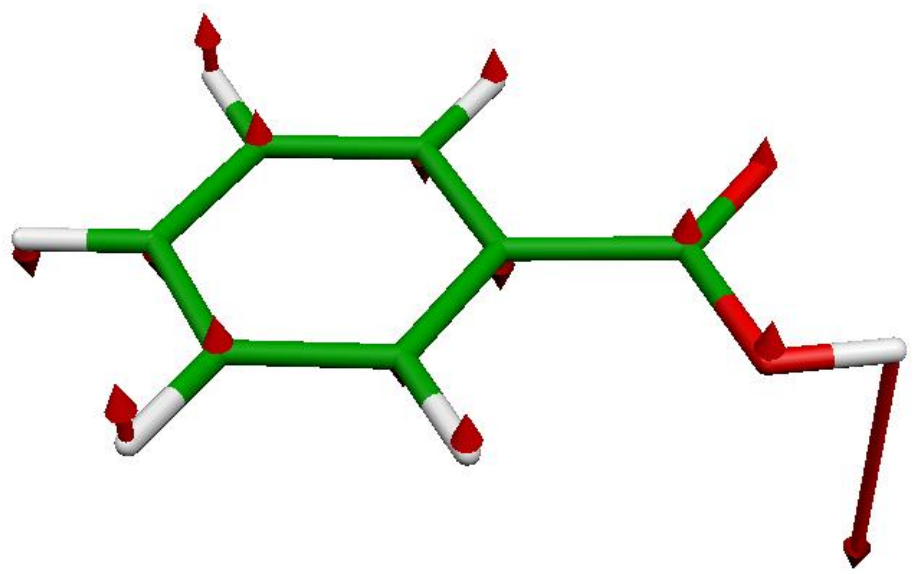


Figure 2b, Antony et al., Journal of Chemical Physics

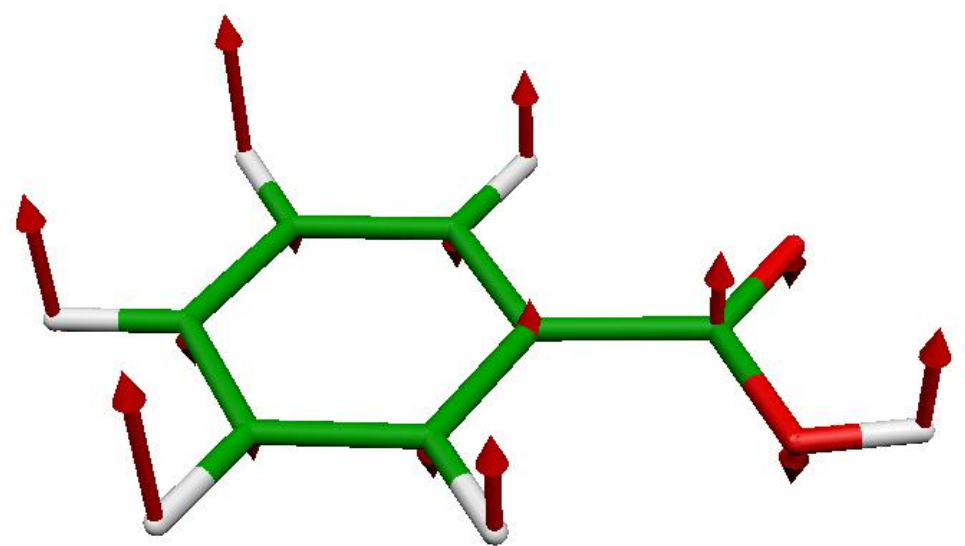


Figure 2c, Antony et al., Journal of Chemical Physics

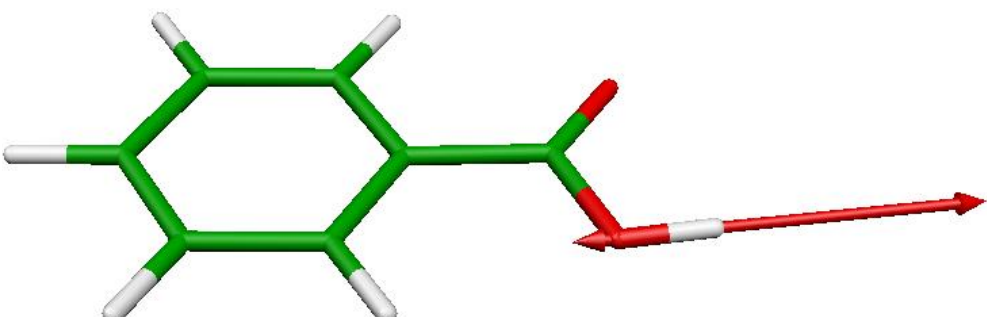


Figure 3a, Antony et al., Journal of Chemical Physics

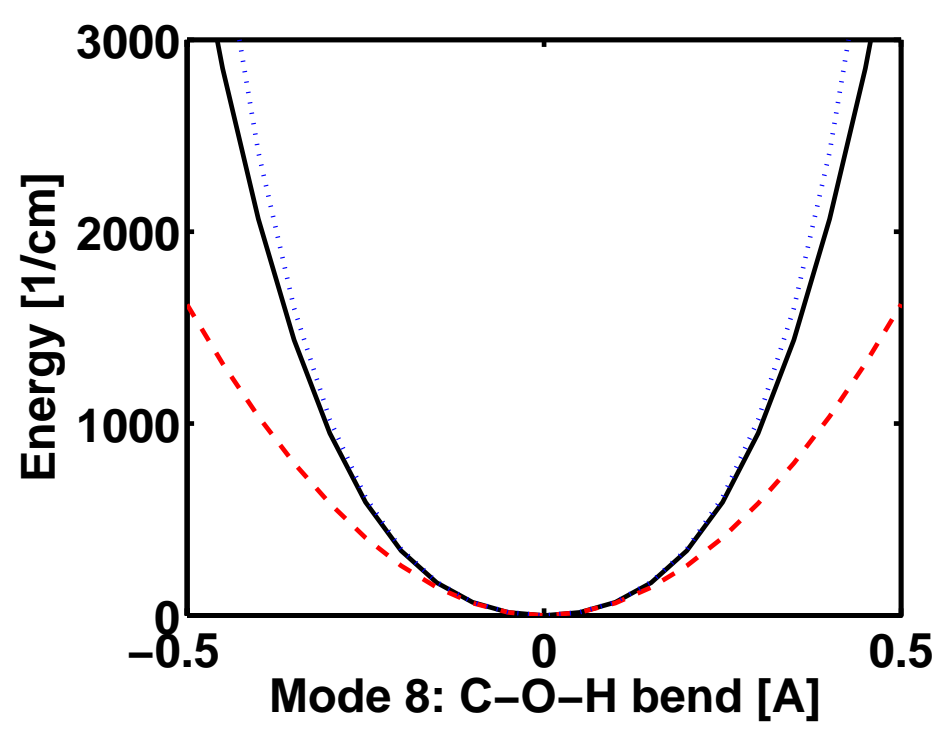


Figure 3b, Antony et al., Journal of Chemical Physics

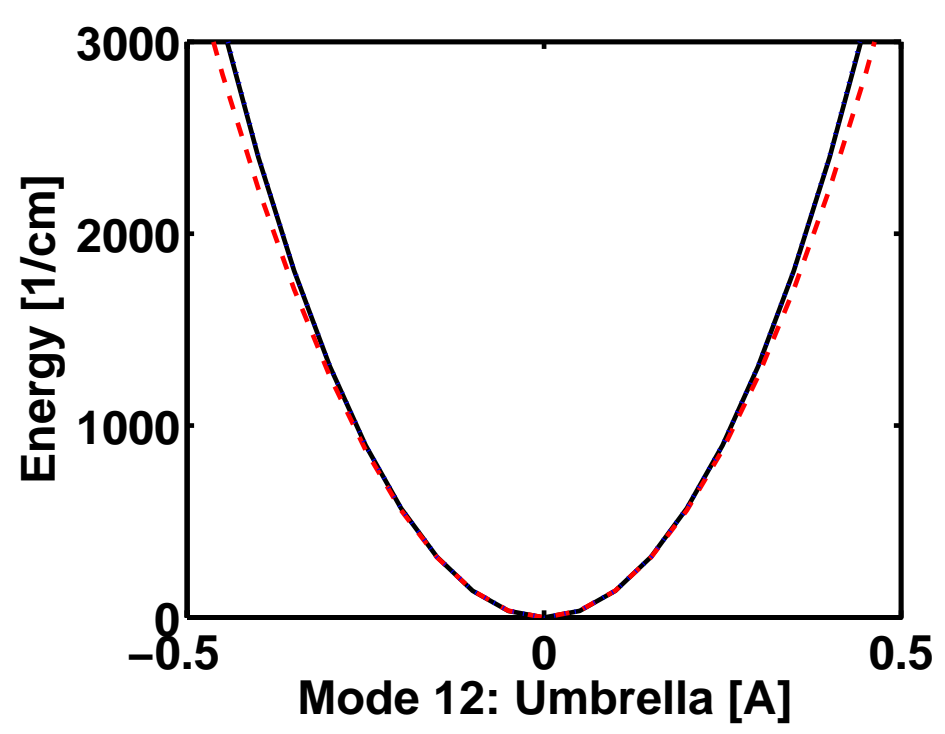


Figure 3c, Antony et al., Journal of Chemical Physics

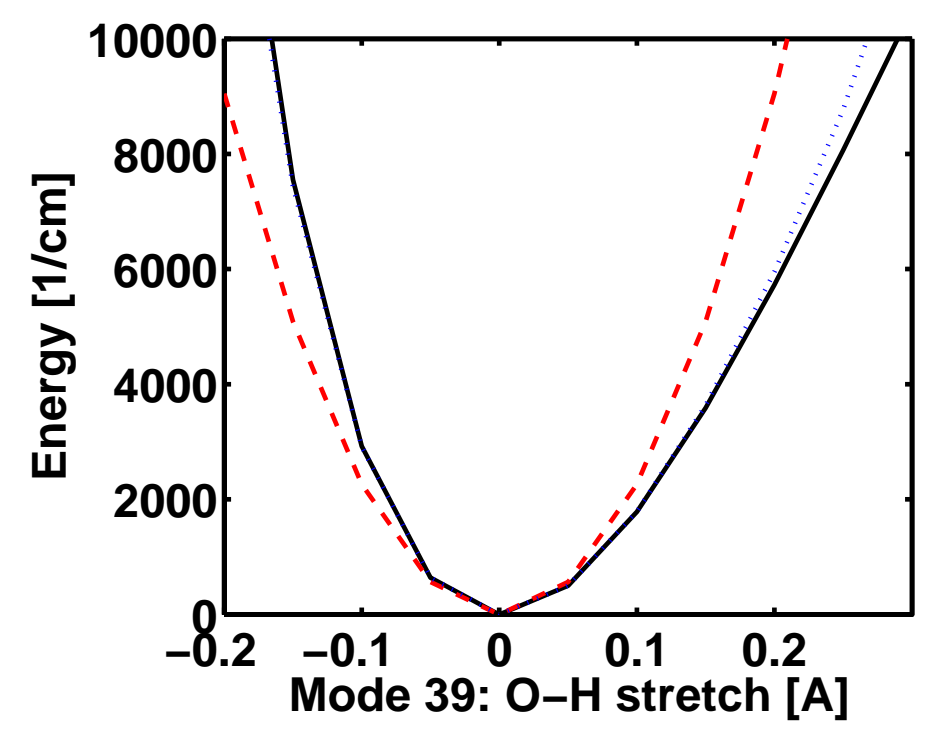


Figure 4a, Antony et al., Journal of Chemical Physics

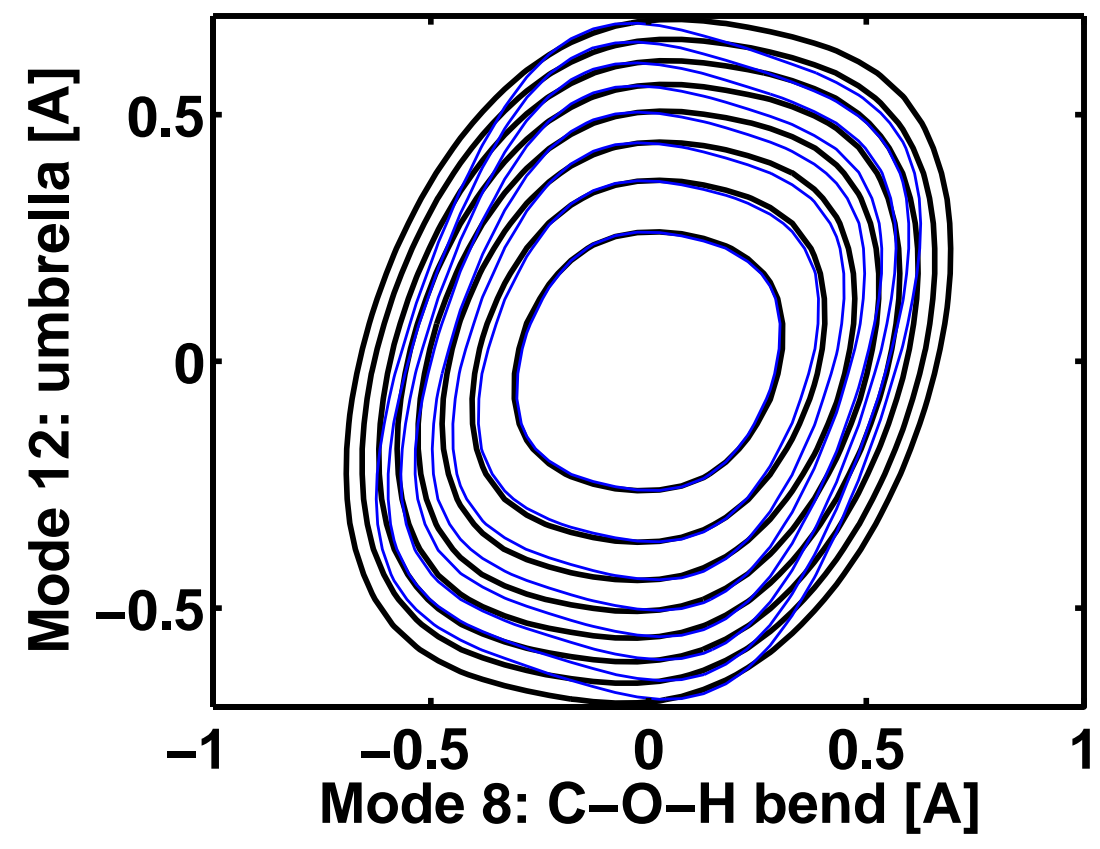


Figure 4b, Antony et al., Journal of Chemical Physics

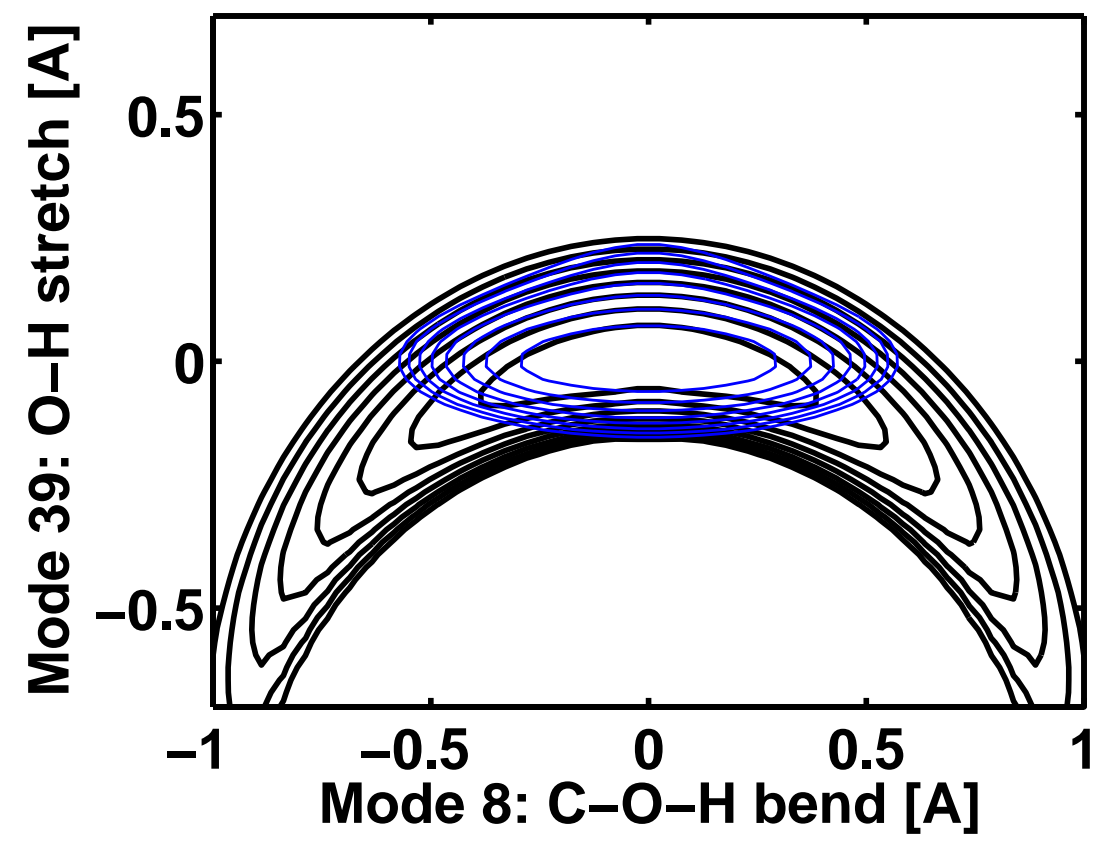


Figure 5a, Antony et al., Journal of Chemical Physics

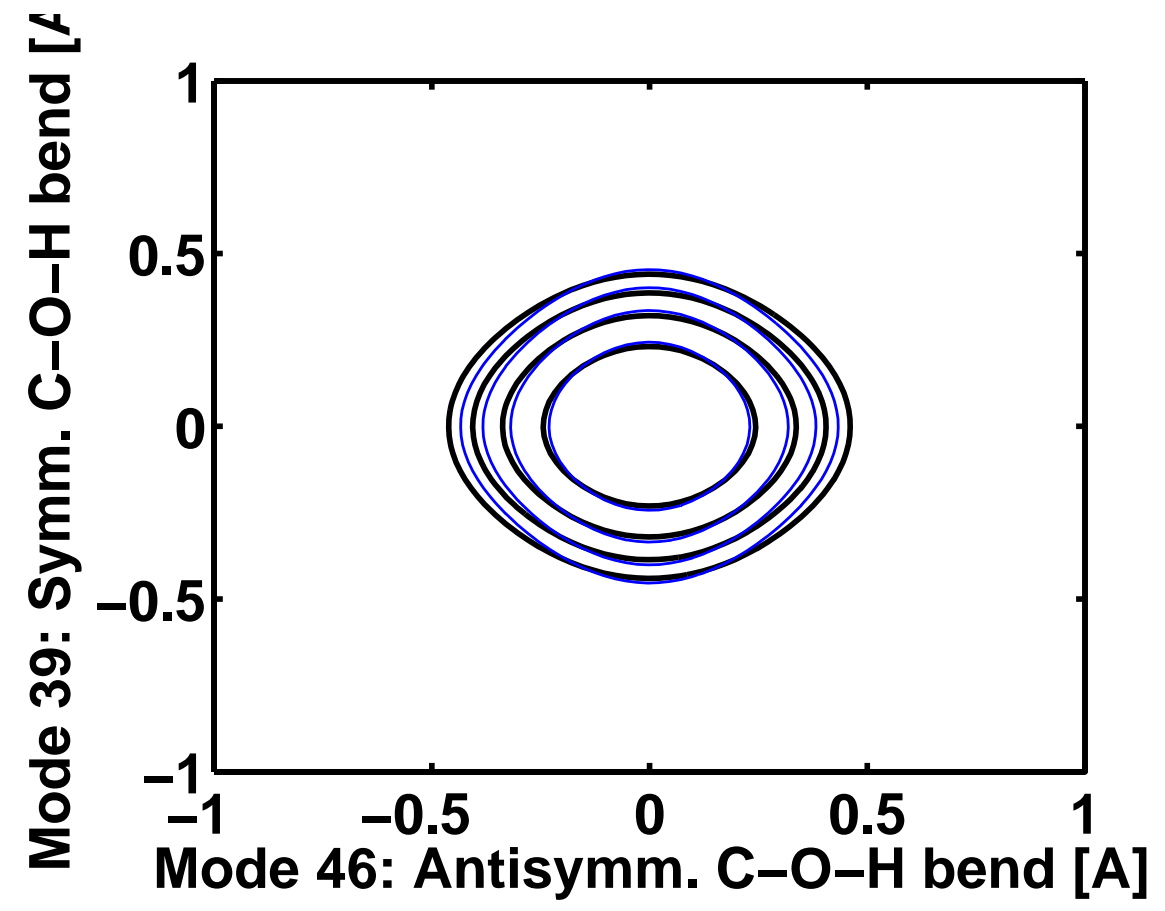


Figure 5b, Antony et al., Journal of Chemical Physics

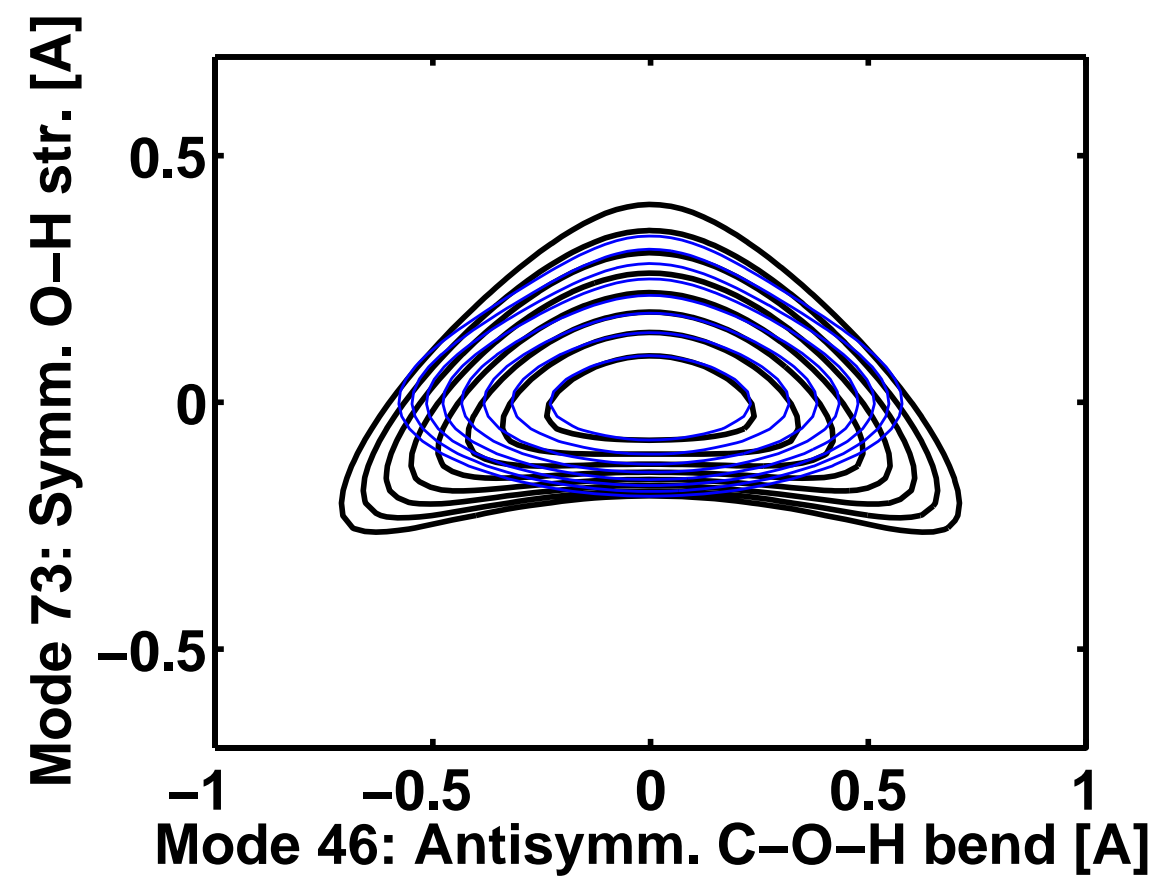


Figure 5c, Antony et al., Journal of Chemical Physics

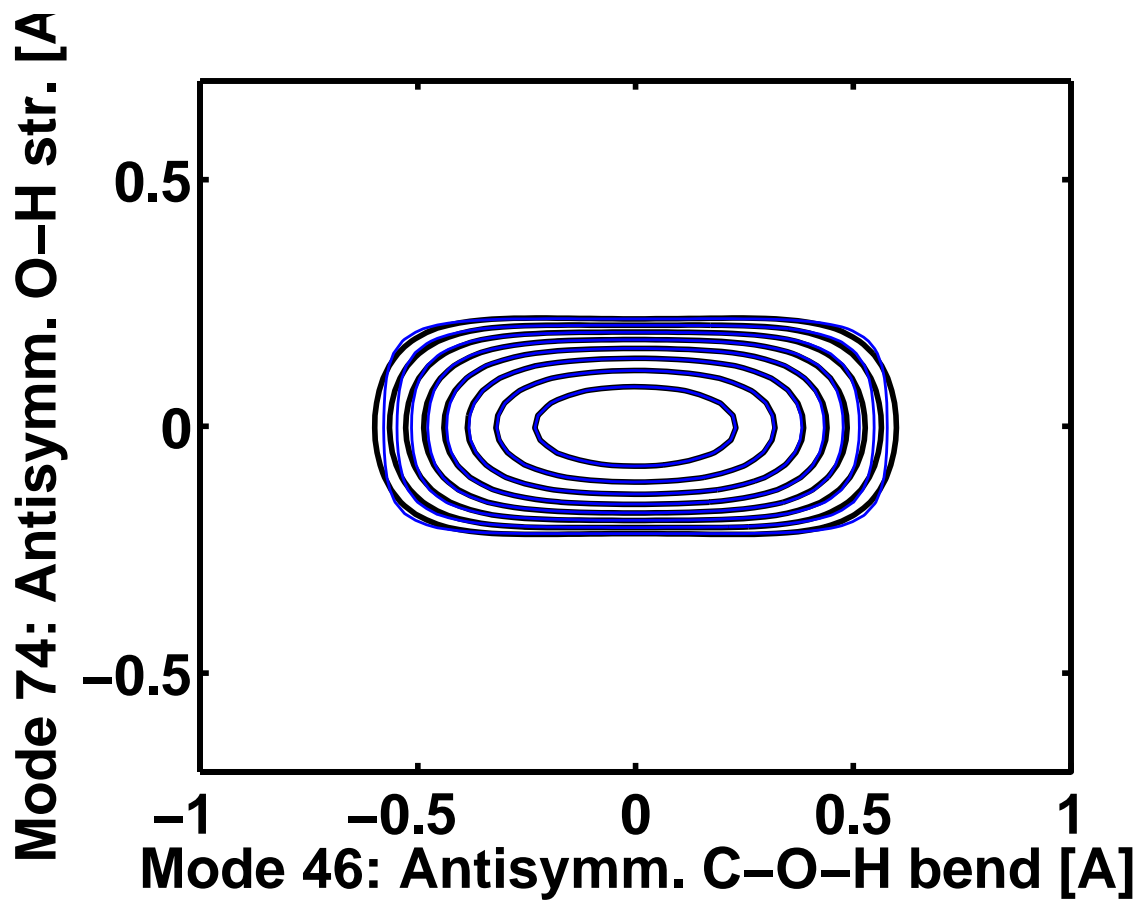

